\title{
EARTHQUAKES ON RIEMANN SURFACES AND ON MEASURED GEODESIC LAMINATIONS
}

\author{
FRANCIS BONAHON
}

\begin{abstract}
Let $S$ be a closed orientable surface of genus at least 2 . We study properties of its Teichmüller space $\mathscr{T}(S)$, namely of the space of isotopy classes of conformal structures on $S$. W. P. Thurston introduced a certain compactification of $\mathscr{T}(S)$ by what he called the space of projective measured geodesic laminations. He also introduced some transformations of Teichmüller space, called earthquakes, which are intimately related to the geometry of $\mathscr{T}(S)$. A general problem is to understand which geometric properties of Teichmüller space subsist at infinity, on Thurston's boundary. In particular, it is natural to ask whether earthquakes continuously extend at certain points of Thurston's boundary, and at precisely which points they do so. This is the principal question addressed in this paper.
\end{abstract}

Let us be a little more precise. By the Uniformization Theorem, every conformal structure on the surface $S$ is realized by a unique Riemannian metric of constant curvature $-k^{2}$, for every $k>0$. We can therefore identify the Teichmüller space $\mathscr{T}(S)$ to the space $\mathscr{T}_{k}(S)$ of isotopy classes of Riemannian metrics of constant curvature $-k^{2}$ on $S$. Since there is no reason to prefer one $\mathscr{T}_{k}(S)$ to another, we can take all of them and consider the space $\mathscr{T}_{0, \infty[}(S)=\bigcup_{k>0} \mathscr{T}_{k}(S)$ of isotopy classes of Riemannian metrics of constant curvature on $S$.

Thurston observed that, when the curvature of a metric tends to $-\infty$, this metric behaves more and more like the transverse measure of a measured geodesic lamination on the surface. The precise definition of a measured geodesic lamination is given in $\S 1$. For the sake of this introduction, however, we can just say that a typical example is provided by an isotopy class of simple closed curves on $S$, endowed with a positive weight, and that a general measured geodesic lamination is a kind of diffused version of this example. This observation led Thurston to add to $\mathscr{T}_{0, \infty}(S)$ the space $\mathscr{M} L(S)=\mathscr{T}_{\infty}(S)$ of measured geodesic laminations and to define a topological space $\mathscr{T}_{0, \infty}(S)=$ $\mathscr{T}_{0, \infty[}(S) \cup \mathscr{T}_{\infty}(S)$. The precise definition of the topology of $\mathscr{T}_{10, \infty]}(S)$ is given at the end of $\S 2$. Thurston's compactification of Teichmüller space is obtained as the quotient space of $\mathscr{T}_{j 0, \infty]}(S)$ under the multiplicative action of $\mathbb{R}^{+}$(see [Th1, FLP]).

Received by the editors December 9, 1988 and, in revised form, April 20, 1990.

1980 Mathematics Subject Classification (1985 Revision). Primary 32G15, 57M05.

Key words and phrases. Teichmüller space, measured laminations, earthquakes.

This research was partially supported by NSF grant DMS-8700642 and by a fellowship from the Alfred P. Sloan Foundation. 
An earthquake is a certain 1-parameter family of transformations $E_{\alpha}^{t}$ : $\mathscr{T}(S) \rightarrow \mathscr{T}(S)$, where $t \in \mathbb{R}$ and where $\alpha$ is a measured geodesic lamination on the surface $S$. When $\alpha$ is an isotopy class of simple closed curves, the definition of the corresponding earthquake map $E_{\alpha}^{t}: \mathscr{T}_{k}(S) \rightarrow \mathscr{T}_{k}(S)$ goes back to W. Fenchel and J. Nielsen [FN]. In this case and if $m$ is a Riemannian metric of constant curvature $-k^{2}, E_{\alpha}^{t} m$ is roughly defined as follows (see $\S 1$ for details). Let $\alpha^{*}$ be the (unique) simple closed $m$-geodesic representing $\alpha$. Then the metric $E_{\alpha}^{t} m$ is obtained by transforming $m$ under a diffeomorphism of $S-\alpha^{*}$ which is isotopic to the identity and shifts one side of $\alpha^{*}$ with respect to the other, where the direction and amount of shifting is determined by $t$ and by the weight of $\alpha$. In particular, if $\alpha$ has weight 1 and if $l_{m}(\alpha)$ denotes the $m$-length of the geodesic $\alpha^{*}, E_{\alpha}^{l_{m}(\alpha)} m$ is obtained by transforming $m$ under a full Dehn twist around $\alpha$. Thurston proved that this construction extends to any measured geodesic lamination $\alpha$, in a way which is continuous in $\alpha$ and in $t$ (see [Ke; EM, §3]).

We would like to know to which extent the resulting earthquake map $E_{\alpha}^{t}$ : $\mathscr{T}_{] 0, \infty[}(S) \rightarrow \mathscr{T}_{] 0, \infty[}(S)$ continuously extends to $T_{] 0, \infty]}(S)$.

One of our results is that, for a measured geodesic lamination $\alpha$ and for $t \in \mathbb{R}$, the earthquake map $E_{\alpha}^{t}: \mathscr{T}_{0, \infty[}(S) \rightarrow \mathscr{T}_{] 0, \infty[}(S)$ extends continuously at those points of the boundary $\mathscr{T}_{\infty}(S)$ which correspond to measured geodesic laminations whose support transversely meet all components of the support of $\alpha$. This is proved as Theorem 14 in $\S 4$. As a partial converse, we prove in Proposition 18 that $E_{\alpha}^{t}$ has no continuous extension at the point $\alpha \in \mathscr{T}_{\infty}(S)$.

As observed above, if $\alpha$ is a homotopy class of simple closed curves with weight 1 , the transformation $m \mapsto E_{\alpha}^{l_{m}(\alpha)} m$ of $\mathscr{T}_{0, \infty[}(S)$ is the one induced by a Dehn twist of $S$, and therefore extends continuously to all of $\mathscr{T}_{0, \infty]}(S)$, by naturality of Thurston's topology on $\mathscr{T}_{10, \infty]}(S)$ under diffeomorphisms of $S$. We can therefore expect better continuity properties by a similar renormalization of the time parameter $t$. For this, let us consider the transformation $T_{\alpha}^{t}$ of $\mathscr{T}_{0, \infty[}(S)$ defined by $T_{\alpha}^{t} m=E_{\alpha}^{t l_{m}(\alpha)} m$, which we call the length-normalized earthquake of amplitude $t$ along $\alpha$. The main characteristic of these lengthnormalized earthquakes is that they are projectively well behaved in the sense that, if the metric $\lambda m \in \mathscr{T}_{k / \lambda}(S)$ is obtained by multiplying $m \in \mathscr{T}_{k}(S)$ by a number $\lambda>0$, then $T_{\alpha}^{t} \lambda m=\lambda T_{\alpha}^{t} m$. Because of this property, $T_{\alpha}^{t}$ continuously extends to $\mathscr{T}_{10, \infty)}(S)$ if and only if its restriction to $\mathscr{T}(S)$ continuously extends to Thurston's compactification of Teichmüller space.

Papadopoulos proved in [Pa1] that $T_{\alpha}^{t}$ continuously extends to $\mathscr{T}_{0, \infty}(S)$ when $\alpha$ is a homotopy class of simple closed curves. We prove in Theorem 15 and Proposition 19 that, given a measured geodesic lamination $\alpha$, the lengthnormalized earthquake $T_{\alpha}^{t}$ continuously extends to $\mathscr{T}_{0, \infty]}(S)$ if and only if $\alpha$ is connected. This result comes somewhat as a surprise, since it goes against the common empirical belief that "everything which is true for simple closed curves also holds for measured geodesic laminations." Also, few properties are known to depend on the connectivity of a measured geodesic lamination.

Our strategy to prove our continuity results is the following. First of all, there is a natural extension of the definition of earthquakes to the space of measured geodesic laminations, whose theory has been extensively developed by $\mathrm{A}$. Papadopoulos [Pa1-Pa3]. Thurston's topology on $\mathscr{T}_{0, \infty}(S)$ is defined by a family 
of seminorms $d\left(\mu, \mu^{\prime}\right)=\left|i(\mu, \gamma)-i\left(\mu^{\prime}, \gamma\right)\right|$, where $\gamma$ ranges over all isotopy classes of simple closed curves on $S$ and where $i: \mathscr{T}_{0, \infty]}(S) \times \mathscr{M} \mathscr{L}(S) \rightarrow \mathbb{R}^{+}$ is a certain intersection form. Therefore, to prove the continuity of the earthquake map $E_{\alpha}^{t}$ at $\beta \in \mathscr{T}_{\infty}(S)=\mathscr{M} L(S)$, it suffices to prove that $i\left(E_{\alpha}^{t} \mu, \gamma\right)$ converges to $i\left(E_{\alpha}^{t} \beta, \gamma\right)$ as $\mu$ tends to $\beta$, for every simple closed curve $\gamma$. We will actually obtain some kind of $C^{1}$-continuity by proving continuity properties for the time derivatives $\frac{\partial}{\partial t} i\left(E_{\alpha}^{t} \mu, \gamma\right)$, from which the continuity properties of $i\left(E_{\alpha}^{t} \mu, \gamma\right)$ will immediately follow by integration.

When $m \in \mathscr{T}_{0, \infty[}(S)$ is a metric of constant curvature on $S, i(m, \gamma)$ is the length of the (unique) closed $m$-geodesic isotopic to $\gamma$, and there is a wellknown cosine formula for the derivative $\left.\frac{\partial}{\partial t} i\left(E_{\alpha}^{t} m, \gamma\right)\right|_{t=0}$ (see [Ke; EM, $\S 3$; Wo2]). For instance, when $\alpha$ is a simple closed curve with weight 1 , and if $\alpha^{*}$ and $\gamma^{*}$ denote the closed $m$-geodesics isotopic to $\alpha$ and $\gamma$, respectively, this derivative is equal to the sum of the cosines $\cos _{m}(\alpha, \gamma)(x)$ of the angles formed by $\alpha^{*}$ and $\gamma^{*}$ at all of their intersection points $x \in \alpha^{*} \cap \beta^{*}$. For a measured geodesic lamination $\beta \in \mathscr{T}_{\infty}(S), i(\beta, \gamma)$ is the so-called geometric intersection number of the two measured laminations $\beta$ and $\gamma$. In $\S 3$, we prove a formula for the derivative $\left.\frac{\partial}{\partial t^{+}} i\left(E_{\alpha}^{t} \beta, \gamma\right)\right|_{t=0}$ which is very similar to the above cosine formula and which is expressed in terms of certain "cosine functions" $\cos _{\beta}(\alpha, \gamma)(x)$, taking only the values $+1,-1$, and 0 . (A similar formula was obtained by $\mathrm{A}$. Fathi in [Fa2], although under a less general and less geometric form.)

This transforms our problem into proving continuity properties for the above cosine functions $\cos _{\mu}$ for $\mu \in \mathscr{T}_{\mathrm{j} 0, \infty]}(S)$. These continuity properties are proved in the crucial Lemma 10, and justify the name of "cosine" for the functions $\cos _{\beta}$ which we introduced for a measured geodesic lamination $\beta$. Thurston's original definition of the topology on $\mathscr{T}_{10, \infty]}(S)$, based on length functions, gives some information on the way angles between closed geodesics vary when a sequence of metrics degenerates to a measured geodesic lamination. However, we need more than that to deal with general measured geodesic laminations. It turns out that the analysis of Liouville measures developed in [Bo2] provides exactly the right tool to analyze the behavior of angles between geodesics, and to prove the continuity properties required. In some sense, the present article is the first nontrivial application of the point of view developed in [Bo2].

The proof that the earthquake maps $E_{\alpha}^{t}$ and length-normalized earthquakes $T_{\alpha}^{t}$ are not continuous at some points of $\mathscr{T}_{10, \infty]}(S)$ is done by examples in $\S 5$. The reader will observe that we do not completely determine the set of points where these maps are continuous. Indeed, although we have some additional partial results in the case of simple closed curves, we have been unable to decide the (dis)continuity properties of these functions at some points of $\mathscr{T}_{\infty}(S)$. A typical example of such an open problem occurs for two measured geodesic laminations $\alpha, \beta \in \mathscr{M} L(S)$ which have the same support but whose transverse measures are not proportional; it is likely (and true in some cases) that $E_{\alpha}^{t}$ is not continuous at $\beta \in \mathscr{T}_{\infty}(S)$, but our methods fail to prove this property in general.

As a concluding remark, we extend the cosine formula for $\left.\frac{\partial}{\partial t^{+}} i\left(E_{\alpha}^{t} \mu, \gamma\right)\right|_{t=0}$ with $\mu \in \mathscr{T}_{\mathrm{j} 0, \infty]}(S)$ to the case where $\gamma$ is a measured geodesic lamination, 
or even a geodesic current in the sense of [Bo1, Bo2] (see $\S 6)$. This analysis is motivated by the existence of natural symplectic structures on $\mathscr{T}_{k}(S)$, for $k \in] 0, \infty]$, and proves some continuity properties for these structures.

This paper is greatly indebted to the pioneering work $[\mathrm{Pa} 1-\mathrm{Pa} 3]$ of $\mathrm{A}$. Papadopoulos, who set the foundations of earthquake maps on measured laminations. In a recent preprint $[\mathrm{Pa} 4]$, Papadopoulos independently proved that the length-normalized earthquake $T_{\alpha}^{t}$ is continuous on $\mathscr{T}_{10, \infty]}(S)$ when the lamination $\alpha$ is complete, namely when the complement of its support in $S$ consists of ideal triangles. Our articles are in some sense complementary. On one hand, our results are more precise since they deal with all measured geodesic laminations and since we obtain some tangential information. On the other hand, his approach, based on a parametrization of Teichmüller space by measured laminations cutting $\alpha$ transversely, is susceptible to lead to further applications in a different direction.

We would like to thank the referee for devoting much time to carefully reading the manuscript, and for helpful suggestions of improvements.

\section{EARTHQUAKes IN TEICHMÜLleR SPACE}

Throughout this paper, we will consider a fixed closed oriented surface $S$ of negative Euler characteristic (namely of genus at least 2).

On $S$, let $m$ be a metric of constant negative curvature, and let $\alpha$ be a simple closed curve which is not homotopic to 0 . To these data we associate a family of metrics $E_{\alpha}^{t} m, t \in \mathbb{R}$, well defined up to isotopy, as follows.

Consider the (unique) closed $m$-geodesic $\alpha^{*}$ of $S$ that is isotopic to $\alpha$. Take a tubular neighborhood $U \cong S^{1} \times[-\varepsilon,+\varepsilon]$ of $\alpha^{*}$, parametrized so that $S^{1} \times 0$ is isometrically sent to $\alpha^{*}$ and so that each $z \times[-\varepsilon,+\varepsilon]$ isometrically corresponds to a geodesic arc orthogonal to $\alpha^{*}$. We can also require that, for the orientation of $U$ induced by the orientation of $S$ and for the natural orientations of $S^{1}$ and $[-\varepsilon,+\varepsilon]$, the intersection number of $S^{1} \times 0$ with $z \times[-\varepsilon,+\varepsilon]$ is +1 . Observe that, for this parametrization, the homeomorphism $\left(e^{i \theta}, u\right) \mapsto\left(e^{i(\theta+t)}, u\right)$ of $U \cong S^{1} \times[-\varepsilon,+\varepsilon]$ respects the metric $m$ (because the curvature of $m$ is constant).

Arbitrarily choose a smooth function $\xi:[0, \varepsilon] \rightarrow \mathbb{R}$ which is 1 on a neighborhood of 0 and is 0 on a neighborhood of $\varepsilon$. Then consider the map $\varphi_{t}: S \rightarrow S$ which is the identity on $S-U$ and on $S^{1} \times[-\varepsilon, 0[\subset U$ and which is defined by $\varphi\left(e^{i \theta}, u\right)=\left(e^{i \theta-i t \xi(t)}, u\right)$ on $S^{1} \times[0, \varepsilon] \subset U$. Observe that $\varphi_{t}$ is a diffeomorphism of $S-\alpha^{*}$, and is discontinuous along $\alpha^{*}$. For $t=0, \varphi_{0}$ is of course the identity and, as $t$ increases, an observer sitting on either side of $\alpha^{*}$ sees the other side move to his/her left under the action of $\varphi_{t}$.

Now, consider the metric $\varphi_{t}^{*}(m)$ on $S-\alpha^{*}$ obtained by transforming the metric $m$ by the shear diffeomorphism $\varphi_{t}$. On both sides of $\alpha^{*}$, the metric $\varphi_{t}^{*}(m)$ coincides with the original metric $m$ provided we are sufficiently close to $\alpha^{*}$. In particular, $\varphi_{t}^{*}(m)$ extends to a Riemannian metric over all of $S$, whose curvature is constant and equal to the curvature of $m$. Let $E_{\alpha}^{t} m$ denote this metric on $S$. One readily sees that, up to isotopy, $E_{\alpha}^{t} m$ depends only on $t \in \mathbb{R}$ and on the isotopy classes of $m$ and $\alpha$.

Following Thurston's terminology, we say that $E_{\alpha}^{t} m$ is obtained from $m$ by a (left) earthquake of amplitude $t$ along the curve $\alpha$. 
Observe that, when $t$ is equal to the length $l_{m}(\alpha)$ of the closed geodesic $\alpha^{*}$, $\varphi_{t}$ is continuous and is a diffeomorphism of the whole surface $S$, namely is a Dehn twist around $\alpha$. Therefore, when $t=l_{m}(\alpha), E_{\alpha}^{t} m$ is the familiar metric obtained by transforming $m$ under this Dehn twist.

W. P. Thurston extended this notion to the case where $\alpha$ is a measured geodesic lamination. To define measured geodesic laminations, let us fix a metric $m_{0}$ of negative curvature on $S$. Then, a measured geodesic lamination consists of: a partial foliation of $S$ by $m_{0}$-geodesics, namely a closed subset $\sigma$ of $S$ which is a union of disjoint simple geodesics, and a transverse measure for $\sigma$, namely a measure defined on each arc $k$ transverse to $\sigma$ with support $k \cap \sigma$, and which is invariant under all homotopies of $k$ respecting the leaves of $\sigma$. A typical example is provided by a closed geodesic $\alpha$, equipped with its Dirac (counting) transverse measure; a slight extension of this example is the measured lamination $\lambda \alpha$, obtained by multiplying the above Dirac measure by $\lambda>0$.

It turns out that the notion of measured geodesic lamination is independent of the negatively curved metric $m_{0}$ we chose on $S$ (see [Le, Th2], or the viewpoint developed in [Bo2]). The space $\mathscr{M} L(S)$ has a natural topology, for which two measured geodesic laminations are close if they induce approximately the same measures on finitely many arcs transverse to them. For this topology, the (positive real) multiples of simple closed geodesics are dense in $\mathscr{M} L(S)$.

Thurston proved the following result, whose exposition can be found in [Ke] (compare also [Th3; EM, §3]).

Proposition 1. There is a (unique) continuous map $\mathscr{M} L(S) \times \mathbb{R} \times \mathscr{T}_{1}(S) \rightarrow \mathscr{T}_{1}(S)$, associating an element $E_{\alpha}^{t} m \in \mathscr{T}_{1}(S)$ to $(\alpha, t, m)$, such that $E_{\lambda \alpha}^{t} m=E_{\alpha}^{\lambda t} m$ for. all $\lambda>0$ and all $\alpha \in \mathscr{M} L(S)$ and such that, when $\alpha$ is a closed geodesic, $E_{\alpha}^{t} m$ is obtained from $m$ by the earthquake defined above.

The metric $E_{\alpha}^{t} m$ defined by Proposition 1 is said to be obtained from $m$ by a (left) earthquake of amplitude $t$ along the measured geodesic lamination $\alpha$.

Remember that, when $\alpha$ is a closed curve, we define earthquakes along $\alpha$ for a metric of arbitrary constant negative curvature. In this construction, the restrictions of $E_{\alpha}^{t}$ to $\mathscr{T}_{k}(S)$ and $\mathscr{T}_{1}(S)$ are clearly related by the formula $E_{\alpha}^{t} m=k^{-1} E_{\alpha}^{k t} k m$ for $m \in \mathscr{T}_{k}(S)=k^{-1} \mathscr{T}_{1}(S)$ (multiplying a metric by $\lambda>0$ multiplies the curvature by $\left.\lambda^{-2}\right)$. When $\alpha$ is any measured geodesic lamination, the same formula, combined with Proposition 1, enables us to define earthquake maps $E_{\alpha}^{t}: \mathscr{T}_{k}(S) \rightarrow \mathscr{T}_{k}(S)$ and a continuous map $\mathscr{M} L(S) \times \mathbb{R} \times \mathscr{T}_{\mathrm{j} 0, \infty[}(S) \rightarrow \mathscr{T}_{\mathrm{j} 0, \infty[}(S)$ given by $(\alpha, t, m) \mapsto E_{\alpha}^{t} m$.

We would like to extend the flow $E_{\alpha}^{t}$ of $\mathscr{T}_{0, \infty[}(S)$ to a 1-parameter family of homeomorphisms of $\mathscr{T}_{0, \infty]}(S)=\left(\bigcup_{k>0} \mathscr{T}_{k}(S)\right) \cup \mathscr{M} L(S)$. However, we will see in $\S 5$ that this is not possible, because $E_{\alpha}^{t}$ does not extend continuously to $\mathscr{T}_{0, \infty]}(S)$ at certain points of $\mathscr{T}_{\infty}(S)=\mathscr{M} L(S)$. On the other hand, when $\alpha$ is a simple closed geodesic, we already observed that the map $m \mapsto E_{\alpha}^{l_{m}(\alpha)} m$ is induced by the Dehn twist around $\alpha$; in particular, because Thurston's topology on $\mathscr{T}_{10, \infty]}(S)$ is natural under diffeomorphisms of $S$, this map continuously extends to $\mathscr{T}_{10, \infty]}(S)$. We would like to see whether the same property holds for any measured geodesic lamination $\alpha$. For this, we need the following notion of $m$-length of a measured geodesic lamination $\alpha$. 
Proposition 2. There is a (unique) continuous map associating to $(m, \alpha) \in$ $\mathscr{T}_{0, \infty[}(S) \times \mathscr{M} L(S)$ a number $l_{m}(\alpha) \in\left[0, \infty\left[\right.\right.$, such that $l_{m}(\lambda \alpha)=\lambda l_{m}(\alpha)$ for all $\lambda>0$ and such that, when $\alpha$ is a simple closed curve, $l_{m}(\alpha)$ is the length of the simple closed m-geodesic homotopic to $\alpha$.

Proof. See [Th2; Bo1, §4] or [Bo2].

Here is how $l_{m}(\alpha)$ is defined. Remember that $\mathscr{M} L(S)$ was initially defined by using an (arbitrary) reference metric of negative curvature. Choose $m$ to be this reference metric. On $S$, consider the measure whose support is equal to the support of $\alpha$, and which is locally the product of the $m$-length measure along the leaves of $\alpha$ and of the transverse measure of $\alpha$. Then $l_{m}(\alpha)$ is equal to the total mass of this measure.

Given $\alpha \in \mathscr{M} L(S)$ and $m \in \mathscr{T}_{10, \infty[}(S)$ we can now define the (left) lengthnormalized earthquake $T_{\alpha}^{t} m$ of amplitude $t$ along $\alpha$ by the formula

$$
T_{\alpha}^{t} m=E_{\alpha}^{t l_{m}(\alpha)} m
$$

We will show that $T_{\alpha}^{t}$ continuously extends to $\mathscr{T}_{10, \infty]}(S)$ for any connected measured geodesic lamination $\alpha \in \mathscr{M} L(S)$.

Note that $T_{\alpha}^{t} \lambda m=\lambda T_{\alpha}^{t} m$ and $T_{\lambda \alpha}^{t} m=T_{\alpha}^{\lambda^{2} t} m$ for all $\lambda>0, \alpha \in \mathscr{M} L(S)$, and $m \in \mathscr{T}_{] 0, \infty[}(S)$.

\section{EARTHQUAKES ON MEASURED GEODESIC LAMINATIONS}

If $\alpha$ and $\beta$ are two simple closed geodesics and if $E_{\alpha}^{1} m$ has a limit as $m \in \mathscr{T}_{0, \infty[}(S)$ tends to $\beta \in \mathscr{M} L(S)=\mathscr{T}_{\infty}(S)$, there is a natural notion of what this limit $E_{\alpha}^{1} \beta$ should be. Namely, let $E_{\alpha}^{1} \beta$ be defined by the family of simple closed geodesics homotopic to the components of the closed 1-submanifold of $S$ obtained as follows: Cut $\beta$ alorg $\alpha$, and then connect each open end of $\beta-\alpha$ to the one which is immediately on its right on the other side of $\alpha$. Observe that this also amounts to modifying $\beta$ on one side of $\alpha$ by applying a certain shear transformation moving this side of $\alpha$ to the left of the other side. By convention, $E_{\alpha}^{1} \beta=\beta$ if $\alpha$ and $\beta$ are disjoint or equal.

The same construction, applied to $n$ parallel copies of $\beta$ in lieu of $\beta$, also defines $E_{\alpha}^{1} n \beta$ for $n \in \mathbb{N}$. If we also want the relations $E_{\alpha}^{t+u} \beta=E_{\alpha}^{t} E_{\alpha}^{u} \beta$ and $E_{\alpha}^{t+u} \beta=\lambda^{-1} E_{\alpha}^{\lambda t} \lambda \beta$ to be satisfied, this clearly fixes what $E_{\alpha}^{t} \beta$ should be for all $t \in \mathbb{Q}$ and all simple closed geodesics $\alpha$ and $\beta$.

In [Pa3], A. Papadopoulos gave a construction which extends this $E_{\alpha}^{t} \beta$ to all measured laminations and all $t \in \mathbb{R}$. When $\alpha$ is a simple closed geodesic, he also proved in [Pa1] some continuity properties of $E_{\alpha}^{t}$ on $\mathscr{T}_{10, \infty]}(S)=$ $\left(\bigcup_{k>0} \mathscr{T}_{k}(S)\right) \cup \mathscr{M} L(S)$. This section is devoted to describing these earthquakes on measured laminations.

Papadopoulos' construction makes use of measured foliations, which are in some sense a diffused version of measured geodesic laminations. A measured foliation consists of a topological 1-dimensional foliation of the surface $S$, with a certain number of saddle singularities, together with a transverse measure in the sense we already defined for geodesic laminations. A complete account of measured foliations can be found in [FLP], for instance. The description given below, in terms of rectangle decompositions of the surface, will amply suffice for our purposes. 
A convenient way to describe a measured foliation is to consider a decomposition of $S$ into finitely many euclidean rectangles $R_{i}=\left[a_{i}, b_{i}\right] \times\left[c_{i}, d_{i}\right]$, whose boundaries are decomposed into a "vertical" part $\partial_{v} R_{i}=\left\{a_{i}, b_{i}\right\} \times\left[c_{i}, d_{i}\right]$ and a "horizontal" part $\partial_{h} R_{i}=\left[a_{i}, b_{i}\right] \times\left\{c_{i}, d_{i}\right\}$, such that:

(a) When two rectangles $R_{i}$ and $R_{j}$ meet, their intersection is an arc equal to $\partial_{v} R_{i} \cap \partial_{v} R_{j}$ or to $\partial_{h} R_{i} \cap \partial_{h} R_{j}$ (possibly a corner).

(b) When $R_{i} \cap R_{j}=\partial_{v} R_{i} \cap \partial_{v} R_{j}$, this intersection arc has the same euclidean length in $R_{i}=\left[a_{i}, b_{i}\right] \times\left[c_{i}, d_{i}\right]$ and in $R_{j}=\left[a_{j}, b_{j}\right] \times\left[c_{j}, d_{j}\right]$.

Condition (a) ensures that the foliations of the rectangles $R_{i}=\left[a_{i}, b_{i}\right] \times$ $\left[c_{i}, d_{i}\right]$ by horizontal arcs $\left[a_{i}, b_{i}\right] \times y$ fit together to give a foliation of the surface $S$. Observe that the singularities occur at the points of $S$ where the sum of the angles of the rectangles meeting at $p$ is at least $3 \pi$. (This sum is everywhere a multiple of $\pi$ and is always at least $2 \pi$.)

If $y_{i}$ denotes the second coordinate of $R_{i}=\left[a_{i}, b_{i}\right] \times\left[c_{i}, d_{i}\right]$, then $\left|d y_{i}\right|$ defines a transverse measure for the foliation of $R_{i}$ by horizontal arcs. Condition (b) implies that these transverse measures match up and define a transverse measure for the whole foliation.

Thus, such a decomposition of $S$ into rectangles uniquely determines a measured foliation $S$.

We will also need a notion of partial measured foliation of $S$. This is a measured foliation of a compact subsurface $\Sigma$ of $S$, satisfying the condition that the boundary $\partial \Sigma$ is a union of leaves and that no component of $\partial \Sigma$ is homotopic to 0 in $S$. The case $\Sigma=S$ is perfectly admitted. A partial measured foliation uniquely determines a measured geodesic lamination, as before. Also, a partial measured foliation of support $\Sigma$ can be described by a decomposition of $\Sigma$ into rectangles $R_{i}$ as above, with the additional property that $\partial \Sigma \cap R_{i}=\partial \Sigma \cap \partial_{h} R_{i}$ for each $R_{i}$.

A measured foliation $A$ uniquely determines a measured geodesic lamination $\alpha$ as follows (see [Le, Th2] for details). First of all, for each leaf of $A$ which does not run through a singularity, there is a unique geodesic of $S$ which stays a bounded distance from this leaf. The fact that the leaves of $A$ do not cross each other implies the same property for the corresponding geodesics. It follows that the closure of the union of these geodesics forms a geodesic lamination $\alpha$. To define the transverse measure, consider an arc $k$ transverse to the lamination. The arc $k$ can be subdivided into subarcs $k_{i}$ such that: For each $k_{i}$, there is an arc $k_{i}^{\prime}$ transverse to $A$ such that the geodesics of $\alpha$ meeting $k_{i}$ are precisely those which either correspond to the leaves of $A$ meeting $k_{i}^{\prime}$ or are a limit of such geodesics. Assigning to $k$ the sum of the transverse measures of the $k_{i}^{\prime}$ defines the transverse measure of $\alpha$.

Conversely, every measured geodesic lamination $\alpha$ is obtained in this way from a measured foliation $A$. Moreover, it can be proved that $A$ is unique up to isotopy and certain operations called "Whitehead moves." This leads to a nice "dictionary" between the theory of measured geodesic laminations and that of measured foliations. An interesting case of this correspondence is when $\alpha$ is a simple closed geodesic. Then, if we remove from $A$ the (finitely many) leaves going from singularity to singularity, what is left is an annulus foliated by parallel closed curves, each isotopic to $\alpha$. For the transverse measure of $A$, the width of this annulus is equal to 1 . 
To define $E_{\alpha}^{t} \beta$, Papadopoulos first considers the case where every component of (the support of) $\alpha$ transversely meets (the support of) $\beta$.

In this case, he proves the following fundamental lemma (Proposition 1 of [Pa3]). We can represent $\beta$ by a measured foliation $B$ and $\alpha$ by a partial measured foliation $A$, with support $\Sigma$, which are in transverse position in the following sense: There is a decomposition of $S$ into rectangles $R_{i}=\left[a_{i}, b_{i}\right] \times$ $\left[c_{i}, d_{i}\right]$, with $\Sigma$ a union of such rectangles, such that $B$ is defined by the foliations of all $R_{i}$ by horizontal arcs, with transverse measure $\left|d y_{i}\right|$, while $A$ is defined by the foliations of the $R_{i}$ contained in $\Sigma$ by vertical arcs, with transverse measure $\left|d x_{i}\right|$, where $\left(x_{i}, y_{i}\right)$ denotes the coordinates in $R_{i}$. We also require that the natural orientation of each rectangle $R_{i}$ coincides with the one induced by the orientation of $S$.

On each such rectangle $R_{i}=\left[a_{i}, b_{i}\right] \times\left[c_{i}, d_{i}\right]$, consider the measured foliation which is defined by straight arcs of slope $-t$ and transverse measure $\left|t d x_{i}+d y_{i}\right|$ if $R_{i}$ is contained in $\Sigma$, and horizontal arcs and transverse measure $\left|d y_{i}\right|$ if $R_{i}$ is not in $\Sigma$. The conditions on the $R_{i}$ ensure that these foliations fit together to give a measured foliation $E_{A}^{t} B$ of the surface $S$. Observe that $E_{A}^{t} B$ coincides with $B$ outside of $\Sigma$, and has the same singular points as $B$. Also, $E_{A}^{0} B=B$.

In this setting, namely when each component of $\beta$ transversely meets $\alpha$ and when $A$ and $B$ are associated to $\alpha$ and $\beta$ as above, we define $E_{\alpha}^{t} \beta$ to be the measured geodesic lamination determined by the measured foliation $E_{A}^{t} B$. In the general case, we define $E_{\alpha}^{t} \beta$ to be equal to $E_{\alpha^{\prime}}^{t} \beta$, where $\alpha^{\prime}$ consists of those components of $\alpha$ which transversely meet $\beta$.

If $\alpha$ and $\beta$ are closed geodesics, and if $t \in \mathbb{Q}$, it is not too difficult to check that this $E_{\alpha}^{t} \beta$ coincides with the one we considered at the beginning of this section.

Papadopoulos proves in [Pa3] that $E_{\alpha}^{t} \beta$ is well defined, namely independent of the choice of the measured foliations $A$ and $B$ we used in the construction. We could actually give an alternative proof of this fact in the present article, by using the case of simple closed geodesics and the continuity properties we are going to prove in $\S 4$.

Observe that $E_{\lambda \alpha}^{t} \beta=E_{\alpha}^{\lambda t} \beta$, that $E_{\alpha}^{t} \lambda \beta=\lambda E_{\alpha}^{t / \lambda} \beta$, and that $E_{\alpha}^{t+u} \beta=E_{\alpha}^{t} E_{\alpha}^{u} \beta$ for all $\alpha, \beta \in \mathscr{M} L(S), \lambda>0$, and $t, u \in \mathbb{R}$.

We want to introduce a renormalization of earthquakes similar to the lengthnormalized earthquake defined in $\S 1$. For this we need an analog of the $m$-length $l_{m}(\alpha)$. The right notion here is the geometric intersection number $l_{\beta}(\alpha)=$ $i(\alpha, \beta)$ of two measured geodesic laminations $\alpha$ and $\beta$, defined by the following proposition.

Proposition 3. There is a (unique) continuous function $\mathscr{M} L(S) \times \mathscr{M} L(S) \rightarrow$ $\mathbb{R}^{+}$, associating a number $i(\alpha, \beta)$ to $(\alpha, \beta) \in \mathscr{M} L(S) \times \mathscr{M} L(S)$, such that $i(\alpha, \lambda \beta)=i(\lambda \alpha, \beta)=\lambda i(\alpha, \beta)$ for all $\lambda \in \mathbb{R}^{+}$, and such that, when $\alpha$ and $\beta$ are distinct simple closed geodesics, $i(\alpha, \beta)$ is the number of intersection points of $\alpha$ and $\beta$.

Proof. See [Re, Corollary 1.11] or [Bo1, §4].

Here is how $i(\alpha, \beta)$ is defined. The transverse measure of $\alpha$ induces a measure $\alpha_{g}$ on each leaf $g$ of the support of $\beta$. (By convention, $\alpha_{g}=0$ 
if $g$ is in the support of $\alpha$.) We can then define a measure on $S$ by locally integrating $\alpha_{g}$ with respect to $\beta$. Namely, each point of $S$ has a neighborhood $U \cong[0,1] \times[0,1]$ such that the leaves of $U \cap \beta$ are of type $x \times[0,1]$. The transverse measure of $\beta$ induces a measure on the first factor $[0,1]$. Then, for $K \subset U$,

$$
\alpha \times \beta(K)=\int \alpha_{g(x)}(K \cap(x \times[0,1])) d \beta(x),
$$

where $g(x)$ is the geodesic of $\beta$ containing $x \times[0,1]$ (if any). Then $i(\alpha, \beta)$ is the total mass of $\alpha \times \beta$.

Thurston defined his topology on $\mathscr{T}_{0, \infty]}(S)$ by the property that a sequence $m_{i} \in \mathscr{T}_{0, \infty[}(S)$ converges to $\beta \in \mathscr{T}_{\infty}(S)=\mathscr{M} L(S)$ if and only if, for every simple closed geodesic $\gamma$, the length $l_{m_{i}}(\gamma)$ converges to $l_{\beta}(\gamma)=i(\beta, \gamma)$. Therefore, the function $\mu \mapsto l_{\mu}(\gamma)$ is continuous on $\mathscr{T}_{0, \infty}$ for every simple closed geodesic $\gamma$.

In fact, for any $\alpha \in \mathscr{M} L(S)$, we have the following stronger continuity property.

Proposition 4. The map $(\mu, \alpha) \mapsto i_{\mu}(\alpha)$ is continuous on $\mathscr{T}_{10, \infty]}(S) \times \mathscr{M} L(S)$.

Proof. See [Bo2], together with the continuity of the intersection number of [Bo1, §4], or combine [Re, Corollary 1.11; FLP, §8].

Henceforth, we will interchangeably use the notation $i(\mu, \alpha)=i(\alpha, \mu)=$ $l_{\mu}(\alpha)$ for any $\mu \in \mathscr{T}_{0, \infty]}(S)$ and any $\alpha \in \mathscr{M} L(S)$. (See [Bo2] for an interpretation of $l_{m}(\alpha)$ as an intersection number when $m \in \mathscr{T}_{0, \infty[}(S)$.)

By analogy with $\S 1$, it is now natural to define on $\mathscr{M} L(S)$ the (left) lengthnormalized earthquake of amplitude $t$ along $\alpha$ to be the transformation which associates to $\beta \in \mathscr{M} L(S)$ the measured geodesic lamination

$$
T_{\alpha}^{t} \beta=E_{\alpha}^{t i(\alpha, \beta)} \beta
$$

Having defined $T_{\alpha}^{t}$ on $\mathscr{T}_{0, \infty]}(S)$ and on $\mathscr{T}_{\infty}(S)=\mathscr{M} L(S)$, we will show in $\S 4$ that the transformation $T_{\alpha}^{t}: \mathscr{T}_{10, \infty]}(S) \rightarrow \mathscr{T}_{0, \infty]}(S)$ is continuous for all connected $\alpha \in \mathscr{M} L(S)$ and all $t \in \mathbb{R}$.

\section{DeRIVATIVES OF LENGTH FUNCTIONS}

The main goal of this article is to prove that, given a connected measured geodesic lamination $\alpha$, the transformation $T_{\alpha}^{t}$ is continuous on $\mathscr{T}_{0, \infty]}(S)$. By definition of the topology of $\mathscr{T}_{10, \infty]}(S)$, it suffices to prove that, for any simple closed geodesic $\gamma$, the function $\mu \mapsto i\left(T_{\alpha}^{t} \mu, \gamma\right)$ is continuous. Our strategy will be to consider the derivative $\frac{\partial}{\partial t} i\left(T_{\alpha}^{t} \mu, \gamma\right)$ and to prove that it is a continuous function of $\mu$, in a weak sense.

When $m \in \mathscr{T}_{10, \infty[}(S)$ is a metric of constant curvature, there is a nice formula for $\left.\frac{\partial}{\partial t} i\left(E_{\alpha}^{t} m, \gamma\right)\right|_{t=0}$ which is easily derived from the formula of the first variation (see [Ke, Wo2; EM, $\S 3]$ ). For this, choose $m$ to be the reference metric used to consider geodesic laminations. For each point $x \in S$ where $\gamma$ and $\alpha$ meet transversely, let $\cos _{m}(\gamma, \alpha)(x)$ denote the cosine of the angles formed at $x$ by $\gamma$ and $\alpha$, counted counterclockwise from $\gamma$ to $\alpha$. Then: 
Proposition 5 [Ke; EM, §3]. If $m \in \mathscr{T}_{0, \infty[}(S), \alpha \in \mathscr{M} L(S)$, and $\gamma$ is a simple closed geodesic, then

$$
\left.\frac{\partial}{\partial t} i\left(E_{\alpha}^{t} m, \gamma\right)\right|_{t=0}=\int_{\gamma} \cos _{m}(\gamma, \alpha) d \alpha,
$$

where the integral is taken with respect to the measure induced on $\gamma$ by the transverse measure of $\alpha$. (Observe that $\cos _{m}(\gamma, \alpha)$ is defined on the support of this measure.)

A similar formula holds for $E_{\alpha}^{t} \beta$ when $\beta \in \mathscr{M} L(S)$, in terms of a certain "cosine" function $\cos _{\beta}^{+}(\gamma, \alpha)$ defined as follows. (Compare [Fa2] for an analogous formula.)

Let $P$ be a point where the simple closed geodesic $\gamma$ transversely meets $\alpha$. Lift $\alpha, \beta, \gamma$ to measured geodesic laminations $\widetilde{\alpha}, \widetilde{\beta}, \tilde{\gamma}$ on the universal covering $\widetilde{S}$ of $S$, and let $\widetilde{P} \in \widetilde{S}$ be a point of the preimage of $P$. Let $a$ and $c$ respectively denote the leaves of $\widetilde{\alpha}$ and $\widetilde{\gamma}$ meeting at $\widetilde{P}$. The space of those geodesics $b$ of $\widetilde{S}$ which cut (transversely) both $a$ and $c$ has two components: Given the orientation of $S$ and considering $c$ as an $x$-axis and $a$ as a $y$-axis, there are the geodesics $b$ of "negative slope" and those of "positive slope" (see Figure 1); more precisely, a geodesic $b$ cutting both $c$ and $a$ has positive slope if, counterclockwise, its end points are between the end points of $c$ and those of $a$, and it has negative slope if its end points are between the end points of $a$ and those of $c$. Since the leaves of $\widetilde{\beta}$ are pairwise disjoint, exactly one of the following properties holds:

(i) There is a leaf $b$ of $\widetilde{\beta}$ which simultaneously cuts $a$ and $c$ with positive slope.

(ii) There is a leaf $b$ of $\widetilde{\beta}$ which simultaneously cuts $a$ and $c$ with negative slope.

(iii) No leaf of $\widetilde{\beta}$ cuts both $a$ and $c$, but there is a leaf of $\widetilde{\beta}$ cutting $a$.

(iv) No leaf of $\widetilde{\beta}$ cuts $a$.

Define $\cos _{\beta}^{+}(\gamma, \alpha)(P)$ to be $-1,+1,+1$, or 0 in case (i), (ii), (iii), or (iv), respectively. This definition is clearly independent of the choice of the point
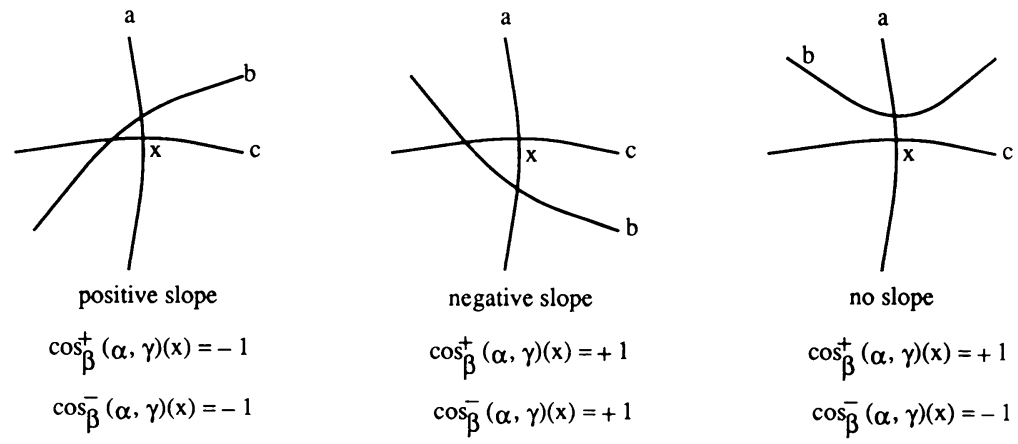

FIGURE 1 
$\widetilde{P}$ in the preimage of $P$. We can also define $\cos _{\beta}^{-}(\gamma, \alpha)(P)$ to be -1 in case (iii) and equal to $\cos _{\beta}^{+}(\gamma, \alpha)(P)$ in the other cases.

Proposition 6. If $\alpha, \beta \in \mathscr{M} L(S)$ and $\gamma$ is a simple closed geodesic, the left and right time derivatives of $i\left(E_{\alpha}^{t} \beta, \gamma\right)$ at $t=0$ are

$$
\begin{aligned}
\left.\frac{\partial}{\partial t^{+}} i\left(E_{\alpha}^{t} \beta, \gamma\right)\right|_{t=0} & =\int_{\gamma} \cos _{\beta}^{+}(\gamma, \alpha) d \alpha, \\
\left.\frac{\partial}{\partial t^{-}} i\left(E_{\alpha}^{t} \beta, \gamma\right)\right|_{t=0} & =\int_{\gamma} \cos _{\beta}^{-}(\gamma, \alpha) d \alpha .
\end{aligned}
$$

The proof of Proposition 6 will take several steps. It will make use of measured foliations, and is analogous to arguments in [Fa1].

First of all, we can restrict attention to the case where $\beta$ transversely meets all components of $\alpha$. Indeed, if $\alpha^{\prime}$ denotes the union of those components of $\alpha$ which transversely meet $\beta$, then $E_{\alpha^{\prime}}^{t} \beta=E_{\alpha}^{t} \beta$ and replacing $\alpha$ by $\alpha^{\prime}$ does not change the integrals in Proposition 6, by definition of the functions $\cos _{\beta}^{+}$ and $\cos _{\beta}^{-}$.

Then, by [Pa3], we can realize $\alpha$ by a partial measured foliation $A$ and $\beta$ by a measured foliation $B$ such that $A$ and $B$ are in transverse position, as described in $\S 2$.

By [FLP, $\S 5], \gamma$ can be homotoped to a curve $\gamma^{\prime}$ which is quasi-transverse to $B$, in the following sense. First of all, $\gamma^{\prime}$ meets the singularities of $B$ at finitely many points. Then each component $k_{i}$ of the complement of the singularities in $\gamma^{\prime}$ is either transverse to $B$ or contained in a leaf of $B$. Lastly, two consecutive such $k_{i}$ are always locally separated by a prong (= leaf leading to a singularity) of the singularity where they meet, unless they are themselves two prongs of the singularity. (See Figure 2 for an example.) It should be observed that $\gamma^{\prime}$ may not necessarily be embedded.

A fundamental property of quasi-transverse curves is that

$$
i(\beta, \gamma)=\int_{\gamma^{\prime}} d B
$$

where $d B$ denotes the transverse measure of $B$ (see [FLP, $\S 5]$ ).

Consider the support $\Sigma$ of $A$. The product of the transverse measure of $A$ and $B$ defines on $\Sigma$ a euclidean metric with singularities at the singularities

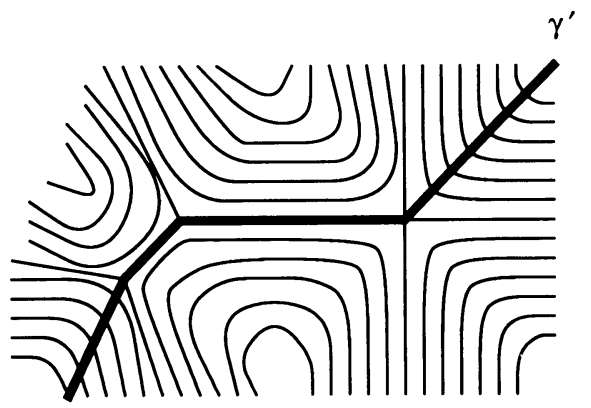

FIGURE 2 
of $A$ (which are also singularities of $B$ ). In other words, this metric is the euclidean metric of the rectangles $R_{i}$ occurring in the definition of "transverse position" in $\S 2$.

Although this metric has singularities it admits a well-defined notion of geodesic. A geodesic consists of arcs joining the singularities, which are straight for the flat metric outside of the singularities. At the singularities, there is a requirement that the angles on either side of a geodesic must be at least $\pi$. The Gauss-Bonnet formula also enables one to define a notion of curvature, which in this case is a Dirac measure concentrated at the singularities; in particular, this curvature is always nonpositive. It easily follows that every arc in $\Sigma$ is homotopic fixing end points to a unique geodesic arc, and that every closed curve which is not homotopic to 0 is homotopic to a closed geodesic; in addition, such a geodesic is either unique or part of an annulus of isotopic geodesics.

Let $\gamma^{\prime \prime}$ be obtained from $\gamma^{\prime}$ by replacing each component of $\Sigma \cap \gamma^{\prime}$ by the geodesic arc (or a closed geodesic if $\gamma^{\prime} \subset \Sigma$ ) homotopic to it. Each component of $\gamma^{\prime \prime} \cap \Sigma$ and of $\gamma^{\prime \prime}-\Sigma$ thus is quasi-transverse to $B$. We might worry that $\gamma^{\prime \prime}$ may not be quasi-transverse to $B$ near $\partial \Sigma$. However, an index argument shows that each component of $\Sigma \cap \gamma^{\prime \prime}$ is homotopic to the corresponding component of $\Sigma \cap \gamma^{\prime}$ through a family of arcs quasi-transverse to $B$ (compare [FLP, $\left.\S 5\right]$ ). It follows that $\gamma^{\prime \prime}$ is quasi-transverse to $B$.

Without loss of generality, we can therefore assume that $\gamma^{\prime}=\gamma^{\prime \prime}$ is quasitransverse to $B$ and is geodesic on $\Sigma$ for the piecewise flat metric defined by $A$ and $B$. In particular, $\Sigma \cap \gamma^{\prime}$ can be subdivided into arcs $k_{i}, \ldots, k_{n}$ which are linear for the flat structure. For each such $k_{i}$, let $a_{i} \in[0, \infty[$ be its $A$-measure and let $b_{i} \in \mathbb{R}$ be defined as follows: $\left|b_{i}\right|$ is the $B$-measure of $k_{i}$, and $b_{i}>0$ if and only if the angle from $B$ to $k_{i}$, counted counterclockwise, is between 0 and $\pi / 2$.

Now, consider the measured foliation $E_{A}^{t} B$ used to define $E_{\alpha}^{t} \beta$ in $\S 2$. Clearly

$$
\int_{\gamma^{\prime}} d E_{A}^{t} B=\int_{\gamma^{\prime}-\Sigma} d B+\sum_{i=1}^{n}\left|t a_{i}+b_{i}\right| .
$$

Lemma 7. For $|t|$ sufficiently small,

$$
\int_{\gamma^{\prime}} d E_{A}^{t} B=i\left(\gamma, E_{\alpha}^{t} \beta\right) .
$$

Proof. Let $k_{i}, a_{i}, b_{i}$ be as above. If $b_{i} \neq 0$ for each $k_{i}$ meeting $\partial \Sigma$, then $\gamma^{\prime}$ is quasi-transverse to $E_{A}^{t} B$ provided $|t|$ is less than all these $\left|b_{i}\right|$. The result then follows from [FLP, $\S 5]$.

In the general case, assume that $0<t<-b_{i}$ for all arcs $k_{i}$ such that $k_{i}$ meets $\partial \Sigma$ and $b_{i}<0$. Then $\gamma^{\prime}$ fails to be quasi-transverse to $E_{\alpha}^{t} \beta$ precisely at those points $x \in \gamma^{\prime} \cap \partial \Sigma$ such that $\gamma^{\prime}$ locally consists of leaves of $B$ near $x$ and such that, if $x$ is a singularity of $B$, no prong of $B$ at $x$ is on the right side of $\gamma^{\prime}$ when $\gamma^{\prime}$ is oriented to leave $\Sigma$.

Starting from such a point $x \in \gamma^{\prime} \cap \partial \Sigma$, follow the corresponding component of $\gamma^{\prime}-\Sigma$ until one of the following happens:

(i) We reach a singularity $y$ of $B$, where at least one prong is on the right-hand side of $\gamma^{\prime}$.

(ii) We reach a point $y$ where $\gamma^{\prime}$ reenters $\Sigma$, and $y$ is not a singularity of $B$ as in (i). 

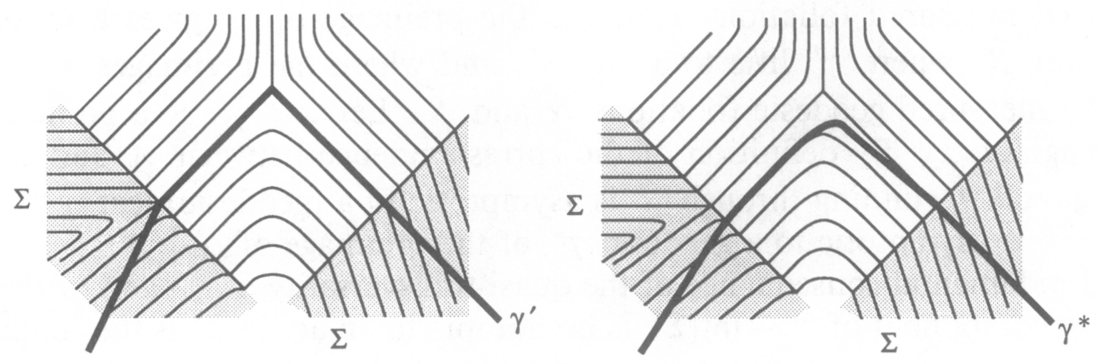

FigURE 3

In both cases, let $k$ denote the subarc of $\gamma^{\prime}$ thus delimited by $x$ and $y$, and let the arc $k^{+}$be the union of $k$ and of a small neighborhood of $x$ in $\gamma^{\prime}$. Then $k^{+}$is homotopic fixing end points to an arc $k^{*}$ transverse to $E_{A}^{t} B$, which has the same $E_{A}^{t} B$-measure as $k^{+}$(see Figure 3 ).

Consider the curve $\left(\gamma^{\prime}-k^{+}\right) \cup k^{*}$. It is now quasi-transverse to $E_{A}^{t} B$ on a neighborhood of $k^{*}$. Indeed, at the point of $\partial k^{*}$ which is close to $x$, a local picture shows that this curve is transverse to $E_{A}^{t} B$ (see Figure 3 ). In case (i), it is quasi-transverse to $E_{A}^{t} B$ at $y$ by definition of quasi-transversality. In case (ii), the crucial observation is that $\gamma^{\prime}$ crosses $E_{A}^{t} B$ in the same direction near both ends of $k^{+}$; it follows that $\left(\gamma^{\prime}-k^{+}\right) \cup k^{*}$ is transverse to $E_{A}^{t} B$ near $y$ in this case (see Figure 3). Thus, replacing $k^{+}$by $k^{*}$ in $\gamma^{\prime}$ gives a curve which has the same $E_{A}^{t} B$-measure as $\gamma^{\prime}$ and which is quasi-transverse to $E_{A}^{t} B$ on a larger portion of the curve.

Performing this process for every point $x$ where $\gamma^{\prime}$ is not quasi-transverse to $E_{A}^{t} B$, we eventually get a curve $\gamma^{*}$ which is homotopic to $\gamma^{\prime}$, is quasi-transverse to $E_{A}^{t} B$, and has the same $E_{A}^{t} B$-measure as $\gamma^{\prime}$. Because $\gamma^{*}$ is homotopic to $\gamma$ and quasi-transverse to $E_{A}^{t} B$, it follows from [FLP, §5] that

$$
i\left(\gamma, E_{\alpha}^{t} \beta\right)=\int_{\gamma^{*}} d E_{A}^{t} B=\int_{\gamma^{\prime}} d E_{A}^{t} B .
$$

This proves Lemma 7.

Combining Lemma 7 with the formula given above the lemma, we get the following formula for the right derivative:

$$
\left.\frac{\partial}{\partial t^{+}} i\left(E_{\alpha}^{t} \beta, \gamma\right)\right|_{t=0}=\sum_{i=1}^{n} a_{i} \frac{b_{i}}{\left|b_{i}\right|},
$$

where by convention $0 / 0=+1$.

In other words, let $c$ be the function on $\gamma^{\prime} \cap \Sigma$ defined by $c(x)=+1$ if $x$ belongs to a $k_{i}$ with $b_{i} \geq 0$ and $c(x)=-1$ otherwise. Then

$$
\left.\frac{\partial}{\partial t^{+}} l\left(E_{\alpha}^{t} \beta, \gamma\right)\right|_{t=0}=\int_{\gamma^{\prime}} c d A .
$$

To conclude the proof of Proposition 6 we need to connect this function $c$ to the "cosine" function $\cos _{\beta}^{+}(\gamma, \alpha)$.

For this, consider a point $x \in \gamma^{\prime}$ such that the leaf of $A$ running through $x$ does not lead to a singularity; in particular, $\gamma^{\prime}$ is transverse to $A$ near $x$. Lift the situation to the universal covering $\widetilde{S}$ of $S$, where the preimage of $A$ gives 
a partial measured foliation $\tilde{A}$, where the preimage of $B$ gives a measured foliation $\widetilde{B}$, where $\gamma^{\prime}$ lifts to a line $\tilde{\gamma}^{\prime}$, and where the preimages of $\alpha$ and $\beta$ give measured geodesic foliations $\tilde{\alpha}$ and $\tilde{\beta}$. Let $\tilde{x} \in \widetilde{\gamma}^{\prime}$ be a point of the preimage of $x$. By definition of the correspondence between $A$ and $\alpha$, the leaf $g_{A}$ of $\tilde{A}$ running through $\tilde{x}$ is asymptotic to a (geodesic) leaf $g_{A}^{*}$ of $\tilde{\alpha}$. Also, $\widetilde{\gamma}^{\prime}$ is asymptotic to a geodesic $\gamma^{*}$ of the preimage of $\gamma$.

A fundamental consequence of the quasi-transversality of $\gamma^{\prime}$ and of the fact that no component of $\gamma^{\prime}-\operatorname{int}(\Sigma)$ is homotopic to an arc in $\Sigma$ is the following:

Lemma 8. The geodesics $g_{A}^{*}$ and $\gamma^{*}$ meet transversely in one point $\tilde{x}^{*}$.

Proof. By an index argument (compare [FLP, §5]), the quasi-transversality of $\gamma^{\prime}$ and the fact that no component of $\gamma^{\prime}-\operatorname{int}(\Sigma)$ is homotopic to an arc in $\Sigma$ imply that $\tilde{\gamma}^{\prime}$ and $g_{A}$ meet only at $\tilde{x}$. Therefore, we only have to show that $\gamma^{*}$ and $g_{A}^{*}$ are not asymptotic.

Because $\gamma^{\prime}$ is transverse to $A$ near $x, \tilde{\gamma}^{\prime}$ also crosses transversely another leaf $h_{A} \neq g_{A}$ of $\tilde{A}$. In addition, the same index argument shows that $\tilde{\gamma}^{\prime}$ and $h_{A}$ meet in exactly one point.

In most cases (see [Le]), the geodesic leaf $h_{A}^{*}$ of $\widetilde{\alpha}$ associated to $h_{A}$ is not asymptotic to $g_{A}^{*}$, which guarantees that $\gamma^{*}$ is not asymptotic to $g_{A}^{*}$.

We therefore only have to worry about the case where $h_{A}^{*}$ and $g_{A}^{*}$ are asymptotic. As proved in [Le], this can occur only when $g_{A}$ projects to a closed leaf of $A$. Then the only way $\tilde{\gamma}^{\prime}$ can be asymptotic to $g_{A}^{*}$ is when $\gamma^{\prime}$ is homotopic to this closed leaf, but this turns out to be incompatible with the fact that $\tilde{\gamma}^{\prime}$ and $g_{A}$ meet in exactly one point. This concludes the proof of Lemma 8.

Therefore, a point $\tilde{x}^{*}$ of intersection of $\widetilde{\alpha}$ and $\gamma^{*}$ is naturally associated to $\tilde{x}$. By equivariance under the action of $\pi_{1}(S)$, it follows that there is a point $x^{*} \in S$ of transverse intersection of $\alpha$ and $\gamma$ which is naturally associated to $x$.

Lemma 9. $c(x)=\cos _{\beta}^{+}(\gamma, \alpha)\left(x^{*}\right)$.

Proof. First consider the case where $\gamma^{\prime}$ is transverse to $B$ near $x$. Then the same argument as in the proof of Lemma 8 shows that the leaf $g_{B}$ of $\widetilde{B}$ running through $\tilde{x}$ is asymptotic to a geodesic leaf $g_{B}^{*}$ of $\widetilde{\beta}$ which transversely meets $\gamma^{*}$. We want to prove that $g_{B}^{*}$ also transversely meets $g_{A}^{*}$.

The two leaves $g_{A}$ and $g_{B}$ meet exactly at $\tilde{x}$, by an index argument as in [FLP, §5].

If $g_{A}$ does not project to a closed leaf of $A$, the same argument as in the proof of Lemma 8 proves that $g_{B}^{*}$ transversely meets $g_{A}^{*}$.

Otherwise, $g_{A}$ projects to a closed leaf $a$ of $A$. Let us follow the projection of $g_{B}$ to $S$ starting from the point $x$. By the Poincaré Recurrence Lemma, this leaf of $B$ must hit $a$ another time. Lifting the situation to $\widetilde{S}$, it follows that $g_{B}$ crosses another lift of $a$, namely another leaf $g_{A}^{\prime}$ of $\tilde{A}$. Observe that $g_{A}^{\prime} \neq g_{A}$ since $g_{B}$ meets $g_{A}$ in only one point. As a consequence, $g_{A}^{\prime}$ is not asymptotic to $g_{A}$, and we conclude as in the proof of Lemma 8 that $g_{B}^{*}$ transversely meets $g_{A}^{*}$.

Therefore, we proved in both subcases that $g_{B}^{*}$ transversely meets $g_{A}^{*}$. In addition, in terms of slopes, the way $g_{B}^{*}$ meets $g_{A}^{*}$ and $\gamma^{*}$ is determined by the way $g_{B}$ meets $g_{A}$ and $\tilde{\gamma}^{\prime}$. By definition of $c(x)$ and of $\cos _{\beta}^{+}(\gamma, \alpha)\left(x^{*}\right)$, it follows that $c(x)=\cos _{\beta}^{+}(\gamma, \alpha)\left(x^{*}\right)$. 
Let us now consider the remaining case, where $\gamma^{\prime}$ coincides with a leaf of $B$ near $x$. Then no leaf of $\widetilde{\beta}$ cuts both $g_{A}^{*}$ and $\gamma^{*}$ : Otherwise, a leaf of $\widetilde{B}$ would meet both $g_{A}$ and $\gamma^{\prime}$, which is easily excluded by an index argument. It follows that $\cos _{\beta}^{+}(\gamma, \alpha)\left(x^{*}\right)=c(x)=+1$ in this case. This concludes the proof of Lemma 9.

Therefore, to each point $x \in \gamma^{\prime}$ which is in a nonsingular leaf of $A$, we have associated a point $x^{*} \in \gamma$, where $\cos _{\beta}^{+}(\gamma, \alpha)\left(x^{*}\right)=c(x)$. The set of such $x$ has full $A$-measure in $\gamma^{\prime}$. Also, by definition of the correspondence between $A$ and $\alpha$, the map $x \mapsto x^{*}$ sends the $A$-measure of $\gamma^{\prime}$ to the $\alpha$-measure of $\gamma$, and the set of such $x^{*}$ has full $\alpha$-measure in $\gamma$. It follows that

$$
\int_{\gamma} \cos _{\beta}^{+}(\gamma, \alpha) d \alpha=\int_{\gamma^{\prime}} c d A
$$

This completes the proof that

$$
\left.\frac{\partial}{\partial t^{+}} i\left(E_{\alpha}^{t} B, \gamma\right)\right|_{t=0}=\int_{\gamma} \cos _{\beta}^{+}(\gamma, \alpha) d \alpha .
$$

The proof that

$$
\left.\frac{\partial}{\partial t^{-}} i\left(E_{\alpha}^{t} B, \gamma\right)\right|_{t=0}=\int_{\gamma} \cos _{\beta}^{+}(\gamma, \alpha) d \alpha
$$

is identical. This concludes the proof of Proposition 6.

\section{CONTINUITY OF LENGTH-NORMALIZED EARTHQUAKES ALONG CONNECTED LAMINATIONS}

This section is devoted to proving that the length-normalized earthquake $T_{\alpha}^{t}$ : $\mathscr{T}_{10, \infty]}(S) \rightarrow \mathscr{T}_{10, \infty]}(S)$ is continuous when the measured geodesic lamination $\alpha$ is connected. By definition of the topology on $\mathscr{T}_{10, \infty]}(S)$, this is equivalent to proving that, for every simple closed geodesic $\gamma$, the function $\mu \mapsto i\left(T_{\alpha}^{t} \mu, \gamma\right)$ is continuous on $\mathscr{T}_{00, \infty]}(S)$. Our strategy will be to prove a stronger continuity property for the derivative of this function with respect to $t$, using the formulas of $\S 3$.

For this, let us introduce some terminology. Let $x$ be a point of intersection of $\alpha$ and $\gamma$. We will say that $\beta$ spans the intersection of $\alpha$ and $\gamma$ at $x$ if the following property holds: In the universal covering $\widetilde{S}$ of $S$, consider the preimages $\widetilde{\alpha}, \widetilde{\beta}$ and $\tilde{\gamma}$ of $\alpha, \beta$, and $\gamma$, respectively, and let $\widetilde{x}$ be a point of the preimage of $x$; then there is a leaf of $\widetilde{\beta}$ which (transversely) cuts the leaves of $\tilde{\alpha}$ and $\tilde{\gamma}$ meeting at $\tilde{x}$.

In particular, if $\beta$ spans the intersection of $\alpha$ and $\gamma$ at $x, \cos _{\beta}^{+}(\gamma, \alpha)(x)=$ $\cos _{\beta}^{-}(\gamma, \alpha)(x)= \pm 1$ according to the way the leaves of $\tilde{\beta}$ cut the leaves of $\tilde{\alpha}$ and $\tilde{\gamma}$ meeting at $\tilde{x}$.

The crucial point in our proof will be the property that, if $\beta$ spans the intersection of $\alpha$ and $\gamma$ at $x$ and if $m \in \mathscr{T}_{10, \infty[}(S)$ tends to $\beta \in \mathscr{T}_{\infty}(S)=$ $\mathscr{M} L(S)$, then $\cos _{m}(\gamma, \alpha)(x)$ converges to $\cos _{\beta}^{+}(\gamma, \alpha)(x)=\cos _{\beta}^{-}(\gamma, \alpha)(x)$. For this, we will use some of the results of [Bo2], which we now review. 
If we endow $S$ with a negatively curved metric $m_{0}$, the space $G(\widetilde{S})$ of geodesics of the universal covering $\widetilde{S}$ of $S$ is, up to homeomorphism, independent of the choice of $m_{0}$. A metric $m \in \mathscr{T}_{k}(S)$ defines a certain measure $L_{m}$, called the Liouville measure, on this space $G(\widetilde{S})$. The basic property of this measure which we will need is the following. Given two geodesics $g$ and $h$ of $\widetilde{S}$ which transversely meet in one point, let ] $g, h$ [ denote the set of geodesics of $\widetilde{S}$ which cut both $g$ and $h$ and whose end points are between those of $g$ and $h$, counterclockwise (namely which have positive slope if $g$ has slope 0 and $h$ has slope $\infty$; compare Figure 1). Then

$$
L_{m}(] g, h[)=-k^{-1} \log \cos ^{2} \theta / 2,
$$

where $\theta \in] 0, \pi[$ is the angle from $g$ to $h$, counted counterclockwise. Observe that

$$
L_{m}(] h, g[)=-k^{-1} \log \sin ^{2} \theta / 2,
$$

and therefore that

$$
\cos \theta=e^{-k L_{m}(] g, h[)}-e^{-k L_{m}(] h, g[)}
$$

and

$$
1=e^{-k L_{m}(] g, h[)}+e^{-k L_{m}(\mathrm{l} h, g[)} .
$$

Similarly, there is a measure $L_{\beta}$ on $G(\widetilde{S})$ which is associated to a measured geodesic lamination $\beta$. If $\widetilde{\beta}$ denotes the preimage of $\beta, L_{\beta}(] g, h[)$ is the $\widetilde{\beta}$-measure of the set of those geodesics of the support of $\widetilde{\beta}$ which cut both $g$ and $h$ and have positive slope, where this $\widetilde{\beta}$-measure is defined by considering suitable arcs transverse to $\widetilde{\beta}$. In particular, $L_{\beta}(] g, h[)$ is positive if and only if a leaf of $\tilde{\beta}$ cuts $g$ and $h$ with positive slope.

A major corollary of [Bo2, Theorem 18] is that the map $\mu \mapsto L_{\mu}$ is continuous on $\mathscr{T}_{10, \infty]}(S)$ if we endow the space of measures on $G(\widetilde{S})$ with the weak* topology.

This is all we are going to need to prove the following crucial lemma.

Lemma 10. Assume that $\beta$ spans the intersection of $\alpha$ and $\gamma$ at $x$. Then $\cos _{m}(\gamma, \alpha)(x)$ converges to $\cos _{\beta}^{+}(\gamma, \alpha)(x)=\cos _{\beta}^{-}(\gamma, \alpha)(x)$ as $m \in \mathscr{T}_{10, \infty[}(S)$ converges to $\beta \in \mathscr{M} L(S)$.

Proof. Lift $\alpha, \beta, \gamma$ to measured geodesic laminations $\widetilde{\alpha}, \widetilde{\beta}, \widetilde{\gamma}$ on the universal covering $\widetilde{S}$ of $S$, and let $\widetilde{x} \in \widetilde{S}$ be a point of the preimage of $x$. Let $g_{\alpha}$ and $g_{\gamma}$ be the leaves of $\widetilde{\alpha}$ and $\tilde{\gamma}$ meeting at $\tilde{x}$.

Since $\beta$ spans the intersection of $\alpha$ and $\gamma$ at $x$, exactly one of $L_{\beta}(] g_{\gamma}, g_{\alpha}[)$ and $L_{\beta}(] g_{\alpha}, g_{\gamma}[)$ is positive. Also

$$
\cos _{\beta}^{+}(\gamma, \alpha)(x)= \begin{cases}-1 & \text { if } L_{\beta}(] g_{\gamma}, g_{\alpha}[)>0, \\ +1 & \text { if } L_{\beta}(] g_{\alpha}, g_{\gamma}[)>0 .\end{cases}
$$

As $m \in \mathscr{T}_{j 0, \infty[}(S)$ tends to $\beta \in \mathscr{M} L(S)=\mathscr{T}_{\infty}(S)$, the measure $L_{m}$ converges to $L_{\beta}$ for the weak* topology. To conclude that $L_{m}(] g_{\gamma}, g_{\alpha}[)$ converges to $L_{\beta}(] g_{\gamma}, g_{\alpha}[)$, we need to check that the $L_{\beta}$-measure of the frontier of $] g_{\gamma}, g_{\alpha}\left[\right.$ is 0 . If this frontier had nonzero $L_{\beta}$-measure, a lift of $\widetilde{\beta}$ would be asymptotic to $g_{\gamma}$ or to $g_{\alpha}$. Since each leaf of a measured geodesic lamination is contained in its set of limit points (see [CB, $\S 3 ;$ FLP, $\S 5]$ ), we would conclude 
that $g_{\gamma}$ or $g_{\alpha}$ is a leaf of $\tilde{\beta}$, contradicting the hypothesis that $\beta$ spans the intersection of $\alpha$ and $\gamma$ at $x$. Therefore, the $L_{\beta}$-measure of the frontier of ] $g_{\gamma}, g_{\alpha}$ [ is 0 , and $L_{m}(] g_{\gamma}, g_{\alpha}[)$ converges to $L_{\beta}(] g_{\gamma}, g_{\alpha}[)$ as $m$ tends to $\beta$.

If $m \in \mathscr{T}_{10, \infty[}(S)$ has curvature $-k_{m}^{2}$, remember that

$$
\cos _{m}(\gamma, \alpha)(x)=e^{-k_{m} L_{m}(] g_{\gamma}, g_{\alpha}[)}-e^{\left.-k_{m} L_{m}(] g_{\alpha}, g_{\gamma} l\right)}
$$

and

$$
e^{-k_{m} L_{m}(] g_{\gamma}, g_{\alpha}[)}+e^{-k_{m} L_{m}(] g_{\alpha}, g_{\gamma}[)}=1
$$

Assume for instance that $L_{\beta}(] g_{\gamma}, g_{\alpha}[)>0$, so that $\cos _{\beta}^{+}(\gamma, \alpha)(x)$ $=-1$. As $m \in \mathscr{T}_{] 0, \infty[}(S)$ tends to $\beta, k_{m}$ converges to $+\infty$. In particular, $k_{m} L_{m}(] g_{\gamma}, g_{\alpha}$ [) converges to $+\infty$. From equality $(* *)$, we conclude that $k_{m} L_{m}(] g_{\gamma}, g_{\alpha}$ l) converges to 0 . It then follows from $(*)$ that $\cos _{m}(\gamma, \alpha)(x)$ converges to $\cos _{\beta}^{+}(\gamma, \alpha)(x)=-1$ as $m \in \mathscr{T}_{10, \infty[}(S)$ tends to $\beta$.

The proof is identical when $L_{\beta}(] g_{\alpha}, g_{\gamma}[)>0$.

Lemma 11. If $\beta$ spans the intersection of $\alpha$ and $\gamma$ at $x$, then $\cos _{\beta^{\prime}}^{+}(\gamma, \alpha)(x)$ and $\cos _{\beta^{\prime}}^{-}(\gamma, \alpha)(x)$ converge to $\cos _{\beta}^{+}(\gamma, \alpha)(x)=\cos _{\beta}^{-}(\gamma, \alpha)(x)$ as $\beta^{\prime} \in$ $\mathscr{M} L(S)$ converges to $\beta$.

Proof. This is an immediate consequence of the definition of the functions $\cos ^{+}$and $\cos ^{-}$.

Proposition 12. Let $\alpha, \beta \in \mathscr{M} L(S)$ and let $\gamma$ be a simple closed geodesic. If $\beta$ spans $\alpha$-almost all intersection points of $\gamma$ and $\alpha$, then the left and right derivatives of $t \mapsto i\left(E_{\alpha}^{t} \mu, \gamma\right)$ at $t=0$ are continuous functions of $\mu \in \mathscr{T}_{\mathrm{j} 0, \infty]}(S)$ at $\beta$.

Proof. Combine the formulas given for these derivatives in Propositions 5 and 6 , Lemmas 10 and 11 , and the theorem of dominated convergence.

Observe that, under the hypothesis of Proposition 12, the left and right derivatives of $t \mapsto i\left(E_{\alpha}^{t} \mu, \gamma\right)$ at $t=0$ coincide if $\mu=\beta$, since $\cos _{\beta}^{+}(\gamma, \alpha)(x)$ $=\cos _{\beta}^{-}(\gamma, \alpha)(x)$ at all points of $\gamma \cap \alpha$ spanned by $\beta$.

Lemma 13. Let $\alpha, \beta \in \mathscr{M} L(S)$ and let $\gamma$ be a simple closed geodesic. Assume that $\beta$ transversely meets all components of $\alpha$. Then, for all but an isolated set of values of $t, E_{\alpha}^{t} \beta$ spans $\alpha$-almost all intersection points of $\gamma$ and $\alpha$.

Proof. Let us go back to the proof of Proposition 6. There, using the hypothesis that $\beta$ cuts all components of $\alpha$, we had realized $\alpha$ by a partial measured foliation $A$ and $\beta$ by a measured foliation $B$, such that $A$ and $B$ were in transverse position. We also observed in the proof of Lemma 9 that, if $\gamma$ can be homotoped to a curve $\gamma^{\prime}$ which is transverse to $B$ on the support $\Sigma$ of $A$ (except at singularities), then $\beta$ spans $\alpha$-almost all intersection points of $\gamma$ and $\alpha$.

In the proof of Lemma 7 , we showed that, for $|t| \neq 0$ sufficiently small, $\gamma$ is homotopic to a curve $\gamma^{*}$ which is quasi-transverse to $A$ and $E_{A}^{t} B$, and is transverse to $E_{A}^{t} B$ on the support of $A$ (except at singularities). It follows that, for $|t| \neq 0$ sufficiently small, $E_{A}^{t} B$ spans $\alpha$-almost all intersection points of $\gamma$ and $\alpha$. 
Since $E_{\alpha}^{t} \beta=E_{\alpha}^{t-u} E_{\alpha}^{u} \beta$, this proves that $E_{\alpha}^{t} \beta$ spans $\alpha$-almost all intersection points of $\gamma$ and $\alpha$ for all but certain isolated values of $t$. (The number of these exceptional values of $t$ can actually be shown to be finite.)

Theorem 14. Let $\alpha$ be a measured geodesic lamination and let $t \in \mathbb{R}$. Then the map which associates $E_{\alpha}^{t} \mu$ to each $\mu \in \mathscr{T}_{10, \infty]}(S)$ is continuous at those $\beta \in \mathscr{M} L(S)$ which transversely meet all components of $\alpha$.

Proof. By definition of the topology of $\mathscr{T}_{10, \infty]}(S)$, it suffices to prove that the function $\mu \mapsto i\left(E_{\alpha}^{t} \mu, \gamma\right)$ is continuous at $\beta$ for every simple closed geodesic $\gamma$.

When $\mu \in \mathscr{T}_{10, \infty[}(S)$, it follows from Proposition 5 that the function $t \mapsto$ $i\left(E_{\alpha}^{t} \mu, \gamma\right)$ has a bounded derivative. In particular,

$$
\begin{aligned}
i\left(E_{\alpha}^{t} \mu, \gamma\right) & =i(\mu, \gamma)+\left.\int_{0}^{t} \frac{\partial}{\partial v} i\left(E_{\alpha}^{v} \mu, \gamma\right)\right|_{v=u} d u \\
& =i(\mu, \gamma)+\int_{0}^{t} \int_{\gamma} \cos _{E_{\alpha}^{u} \mu}(\gamma, \alpha) d \alpha d u
\end{aligned}
$$

by Proposition 5 (since $\left.E_{\alpha}^{u} \mu=E_{\alpha}^{v-u} E_{\alpha}^{u} \mu\right)$.

When $\mu \in \mathscr{T}_{\infty}(S)=\mathscr{M} L(S)$, Lemma 7 shows that the function $t \mapsto i\left(E_{\alpha}^{t} \mu, \gamma\right)$ is locally convex. By Proposition 6, it follows that

$$
\begin{aligned}
i\left(E_{\alpha}^{t} \mu, \gamma\right) & =i(\mu, \gamma)+\left.\int_{0}^{t} \frac{\partial}{\partial v^{+}} i\left(E_{\alpha}^{v} \mu, \gamma\right)\right|_{v=u} d u \\
& =i(\mu, \gamma)+\int_{0}^{t} \int_{\gamma} \cos _{E_{\alpha}^{\mu} \mu}^{+}(\gamma, \alpha) d \alpha d u .
\end{aligned}
$$

To unify the notation, define $\cos _{\mu}^{+}=\cos _{\mu}^{-}=\cos _{\mu}$ when $\mu \in \mathscr{T}_{10, \infty[}(S)$. Then, by Lemmas 10,11, and 13, the function

$$
\mu \mapsto \cos _{E_{\alpha}^{\mu} \mu}^{+}(\gamma, \alpha)(x)
$$

defined on $\mathscr{T}_{10, \infty)}(S)$ is continuous at $\beta$ for all but finitely many values of $u \in[0, t]$ and for $\alpha$-almost all $x \in \gamma$. By dominated convergence,

$$
\mu \mapsto \int_{\gamma} \cos _{E_{\alpha}^{\mu} \mu}^{+}(\gamma, \alpha) d \alpha
$$

is continuous at $\beta$ for all but finitely many values of $u \in[0, t]$. Another application of dominated convergence gives that

$$
\mu \mapsto \int_{0}^{t} \int_{\gamma} \cos _{E_{\alpha}^{u} \mu}^{+}(\gamma, \alpha) d \alpha d u=i\left(E_{\alpha}^{v} \mu, \gamma\right)-i(\mu, \gamma)
$$

is continuous at $\beta$. By continuity of the intersection number (Proposition 4), this concludes the proof of Theorem 14.

Theorem 15. Let $\alpha$ be a connected measured geodesic lamination. Then, for any $t \in \mathbb{R}$, the length-normalized earthquake $\mu \mapsto T_{\alpha}^{t} \mu$ is continuous on $\mathscr{T}_{10, \infty]}(S)$. Proof. The continuity at $m \in \mathscr{T}_{] 0, \infty[}(S)$ comes from Propositions 1 and 2 (and is proved in [Ke; EM, §3]).

Since $\alpha$ is connected, Theorem 14 shows that

$$
\mu \mapsto T_{\alpha}^{t} \mu=E_{\alpha}^{t i(\alpha, \mu)} \mu=i(\alpha, \mu) E_{\alpha}^{t} \frac{\mu}{i(\alpha, \mu)}
$$


is continuous at those $\beta \in \mathscr{M} L(S)$ which transversely meet $\alpha$, namely those $\beta$ with $i(\alpha, \beta) \neq 0$.

Thus, we only need to check the continuity at $\beta \in \mathscr{M} L(S)$ with $i(\alpha, \beta)=0$. In this case, we will make use of the following estimate, borrowed from [Pa1].

Lemma 16. If $m \in \mathscr{T}_{] 0, \infty[}(S), \alpha \in \mathscr{M} L(S)$, and $\gamma$ is a simple closed geodesic, then

$$
\left|i\left(E_{\alpha}^{t} m, \gamma\right)-i(m, \gamma)\right| \leq|t| i(\alpha, \gamma)
$$

and

$$
\left|i\left(T_{\alpha}^{t} m, \gamma\right)-i(m, \gamma)\right| \leq|t| i(\alpha, m) i(\alpha, \gamma) .
$$

Proof of Lemma 16. See [Pa1]. Since it is relatively simple, here is a sketch of this proof for the sake of completeness. First consider the case when $\alpha$ is a simple closed geodesic. Then considering the image of $\gamma$ under the shear map used to construct $E_{\alpha}^{t} m$ together with suitable arcs in $\alpha$, one easily constructs a curve $\gamma^{\prime}$ homotopic to $\gamma$ whose $E_{\alpha}^{t} m$-length is $i(m, \gamma)+|t| i(\alpha, \gamma)$. It follows that $i\left(E_{\alpha}^{t} m, \gamma\right) \leq i(m, \gamma)+|t| i(\alpha, \gamma)$ when $\alpha$ is a simple closed geodesic. This inequality holds for a general $\alpha \in \mathscr{M} L(S)$ by continuity. The same argument shows that $i(m, \gamma)=i\left(E_{\alpha}^{-t} E_{\alpha}^{t} m, \gamma\right) \leq i\left(E_{\alpha}^{t} m, \gamma\right)+|t| i(\alpha, \gamma)$. Combining these two inequalities, we get the first statement of the lemma. The second statement is an immediate consequence of the first one.

Observe that the second estimate of Lemma 16 extends to $\mathscr{M} L(S)$, namely that

$$
\left|i\left(T_{\alpha}^{t} \mu, \gamma\right)-i(\mu, \gamma)\right|<|t| i(\alpha, \gamma) i(\alpha, \mu)
$$

for all $\mu \in \mathscr{T}_{10, \infty]}(S)$ and every simple closed geodesic $\gamma$. Indeed, this inequality is clear at $\mu \in \mathscr{M} L(S)$ with $i(\alpha, \mu)=0$, since $T_{\alpha}^{t} \mu=\mu$ in this case; for $\mu \in \mathscr{M} L(S)$ with $i(\alpha, \mu) \neq 0$, it follows from the continuity property of $T_{\alpha}^{t}$ at $\mu$ which we just proved.

Now, as $\mu \in \mathscr{T}_{10, \infty]}(S)$ tends to $\beta \in \mathscr{M} L(S)$ with $i(\alpha, \beta)=0, i(\alpha, \mu)$ converges to $i(\alpha, \beta)=0$. The above estimate then shows that $i\left(T_{\alpha}^{t} \mu, \gamma\right)$ has the same limit as $i(\mu, \gamma)$, namely $i(\beta, \gamma)=i\left(T_{\alpha}^{t} \beta, \gamma\right)$, for every simple closed geodesic $\gamma$. It follows that $T_{\alpha}^{t} \mu$ converges to $T_{\alpha}^{t} \beta$. This concludes the proof of Theorem 15.

\section{DisCONTINUITIES OF EARTHQUAKES AND LENGTH-NORMALIZED EARTHQUAKES}

This section is devoted to proving converses to Theorems 14 and 15 .

First we show that, for any $\alpha \in \mathscr{M} L(S)$, the earthquake map $E_{\alpha}^{t}$ is not continuous on $\mathscr{T}_{0, \infty]}(S)$. This requires a preparatory lemma.

Lemma 17. For every $m \in \mathscr{T}_{] 0, \infty]}(S)$ and every $\alpha \in \mathscr{M} L(S)$,

$$
\lim _{t \rightarrow \pm \infty} \frac{1}{|t|} E_{\alpha}^{t} m=\alpha
$$

Proof. By definition of the topology of $\mathscr{T}_{0, \infty]}(S)$, it suffices to prove that

$$
\lim _{t \rightarrow \pm \infty} \frac{1}{|t|} i\left(E_{\alpha}^{t} m, \gamma\right)=i(\alpha, \gamma)
$$

for every simple closed geodesic $\gamma$. 
By Proposition 5,

$$
\left.\frac{\partial}{\partial u} i\left(E_{\alpha}^{u} m, \gamma\right)\right|_{u=t}=\int_{\gamma} \cos _{E_{\alpha}^{t} m}(\gamma, \alpha) d \alpha .
$$

For each point $x$ of the transverse intersection of $\gamma$ and $\alpha$, (the proof of) Lemma 3.6 of $[\mathrm{Ke}]$ shows that the map

$$
t \mapsto \cos _{E_{\alpha}^{t} m}(\gamma, \alpha)(x)
$$

is strictly increasing and converges to +1 as $t$ tends to $+\infty$. If follows that the function

$$
\left.t \mapsto \frac{\partial}{\partial u} i\left(E_{\alpha}^{u} m, \gamma\right)\right|_{u=t}
$$

is nondecreasing and, by monotone (or dominated) convergence, that

$$
\left.\lim _{t \rightarrow+\infty} \frac{\partial}{\partial u} i\left(E_{\alpha}^{u} m, \gamma\right)\right|_{u=t}=\int_{\gamma} d \alpha=i(\alpha, \gamma) \text {. }
$$

As a consequence, the function $t \mapsto i\left(E_{\alpha}^{t} m, \gamma\right)$ is convex and asymptotic to a linear function $t \mapsto t i(\alpha, \gamma)+C$ as $t$ tends to $+\infty$, for some constant $C$. It immediately follows that

$$
\lim _{t \rightarrow+\infty} \frac{1}{t} i\left(E_{\alpha}^{t} m, \gamma\right)=i(\alpha, \gamma)
$$

A similar argument proves that

$$
\lim _{t \rightarrow-\infty} \frac{1}{|t|} i\left(E_{\alpha}^{t} m, \gamma\right)=i(\alpha, \gamma) \text {. }
$$

Since the above properties hold for all simple closed geodesics $\gamma$, this completes the proof of Lemma 17.

As an immediate application, we get

Proposition 18. If $\alpha \in \mathscr{M} L(S)-0$ and $t \neq 0$, the earthquake map $E_{\alpha}^{t}$ : $\mathscr{T}_{10, \infty]}(S) \rightarrow \mathscr{T}_{\mathrm{j} 0, \infty]}(S)$ is not continuous at $\alpha$.

Proof. Fix $m \in \mathscr{T}_{\mathrm{j} 0, \infty[}(S)$. For $u>0$, set

$$
m_{u}=\frac{1}{u} E_{\alpha}^{t u} m \quad \text { and } \quad m_{u}^{\prime}=\frac{1}{u} E_{\alpha}^{-t u} m .
$$

By Lemma 17,

$$
\lim _{u \rightarrow+\infty} m_{u}=\lim _{u \rightarrow+\infty} m_{u}^{\prime}=\alpha .
$$

On the other hand,

$$
\begin{aligned}
\lim _{u \rightarrow+\infty} E_{\alpha}^{t} m_{u} & =\lim _{u \rightarrow+\infty} E_{\alpha}^{t}\left(\frac{1}{u} E_{\alpha}^{t u} m\right)=\lim _{u \rightarrow+\infty} \frac{1}{u} E_{\alpha}^{t u} E_{\alpha}^{t u} m \\
& =\lim _{u \rightarrow+\infty} \frac{1}{u} E_{\alpha}^{2 t u} m=2|t| \alpha
\end{aligned}
$$

by Lemma 17 . Similarly,

$$
\begin{aligned}
\lim _{u \rightarrow+\infty} E_{\alpha}^{t} m_{u}^{\prime} & =\lim _{u \rightarrow+\infty} E_{\alpha}^{t}\left(\frac{1}{u} E_{\alpha}^{-t u} m\right)=\lim _{u \rightarrow+\infty} \frac{1}{u} E_{\alpha}^{t u} E_{\alpha}^{-t u} m \\
& =\lim _{u \rightarrow+\infty} \frac{1}{u} m=0 .
\end{aligned}
$$


Since these two limits are distinct, this proves that $E_{\alpha}^{t}$ is not continuous at $\alpha$.

Observe that we even proved something stronger: The homeomorphism $E_{\alpha}^{t}$ of $\mathscr{T}_{\mathrm{j} 0, \infty[}(S)$ does not extend continuously to $\mathscr{T}_{0, \infty]}(S)$ (no matter how we define $E_{\alpha}^{t}$ on $\left.\mathscr{M} L(S)\right)$.

Similarly, we get the following converse to Theorem 15.

Proposition 19. For $t \neq 0$, the length-normalized earthquake $T_{\alpha}^{t}$ continuously extends to $\mathscr{T}_{\mathrm{j} 0, \infty \mathrm{l}}(S)$ if and only if $\alpha$ is connected.

Proof. The "if" part is just Theorem 15.

Conversely, assume that $\alpha$ is not connected, let $\alpha_{1}$ be a component of $\alpha$ and let $\alpha_{2}=\alpha-\alpha_{1} \neq \varnothing$. We can always choose $\alpha_{1}$. so that it is not a closed geodesic homotopic to an end of $S-\alpha_{2}$. Then there is a measured geodesic lamination $\beta_{1}$ which is disjoint from $\alpha_{2}$ and such that $i\left(\alpha_{1}, \beta_{1}\right)=t^{-1}$, assuming $t>0$ without loss of generality. (For instance, we can take $\beta_{1}$ to be a simple closed geodesic crossing $\alpha_{1}$ and endowed with a suitable weight.) Set $\beta=\beta_{1} \cup \alpha_{2}$.

Arbitrarily fix $m \in \mathscr{T}_{] 0, \infty[}(S)$ and, for $u>0$, define

$$
m_{u}=\frac{1}{u} E_{\beta}^{u} m=E_{\beta}^{1}\left(\frac{1}{u} m\right)
$$

and

$$
m_{u}^{\prime}=\frac{1}{u} E_{\beta}^{-u} m=E_{\beta}^{-1}\left(\frac{1}{u} m\right) \text {. }
$$

By Lemma 17,

$$
\lim _{u \rightarrow+\infty} m_{u}=\lim _{u \rightarrow+\infty} m_{u}^{\prime}=\beta
$$

Now consider

$$
\begin{aligned}
T_{\alpha}^{t} m_{u} & =E_{\alpha}^{t i\left(\alpha, m_{u}\right)} E_{\beta}^{1}\left(\frac{1}{u} m\right) \\
& =E_{\alpha_{1}}^{t i\left(\alpha, m_{u}\right)} E_{\alpha_{2}}^{t i\left(\alpha, m_{u}\right)} E_{\beta_{1}}^{1} E_{\alpha_{2}}^{1}\left(\frac{1}{u} m\right) \\
& =E_{\alpha_{1}}^{t i\left(\alpha, m_{u}\right)} E_{\alpha_{2}}^{t i\left(\alpha, m_{u}\right)+1} E_{\beta_{1}}^{1}\left(\frac{1}{u} m\right) \\
& =E_{\alpha_{1}}^{t i\left(\alpha, m_{u}\right)}\left(\frac{1}{u} E_{\alpha_{2}}^{t u i\left(\alpha, m_{u}\right)+u} E_{\beta_{1}}^{u} m\right) .
\end{aligned}
$$

As $u$ tends to $+\infty, i\left(\alpha, m_{u}\right)$ converges to $i(\alpha, \beta)=t^{-1}$. By Lemma 16 , it follows that

$$
\begin{aligned}
& \left|i\left(\frac{1}{u} E_{\alpha_{2}\left(\alpha, m_{u}\right)+u}^{t u i} E_{\beta_{1}}^{u} m, \gamma\right)-i\left(\frac{1}{u} E_{\alpha_{2}}^{2 u} E_{\beta_{1}}^{u} m, \gamma\right)\right| \\
& \quad \leq \frac{1}{u}\left|t u i\left(\alpha, m_{u}\right)+u-2 u\right| i\left(\gamma, \alpha_{2}\right) \\
& \quad \leq\left|t i\left(\alpha, m_{u}\right)-1\right| i\left(\gamma, \alpha_{2}\right) \rightarrow 0
\end{aligned}
$$

for every simple closed geodesic $\gamma$. Therefore,

$$
\begin{aligned}
\lim _{u \rightarrow+\infty} & \frac{1}{u} E_{\alpha_{2}}^{t u i\left(\alpha, m_{u}\right)+u} E_{\beta_{1}}^{u} m \\
= & \lim _{u \rightarrow+\infty} \frac{1}{u} E_{\alpha_{2}}^{2 u} E_{\beta_{1}}^{u} m=\lim _{u \rightarrow+\infty} \frac{1}{u} E_{2 \alpha_{2}}^{u} E_{\beta_{1}}^{u} m=2 \alpha_{2} \cup \beta_{1}
\end{aligned}
$$

by Lemma 17 . 
Since $\alpha_{1}$ is connected and transversely meets $2 \alpha_{2} \cup \beta_{1}$, it follows from Theorem 14 that

$$
\begin{aligned}
\lim _{u \rightarrow+\infty} T_{\alpha}^{t} m_{u} & =\lim _{u \rightarrow+\infty} E_{\alpha_{1}}^{t i\left(\alpha, m_{u}\right)}\left(\frac{1}{u} E_{\alpha_{2}}^{t u i\left(\alpha, m_{u}\right)+u} E_{\beta_{1}}^{u} m\right) \\
& =\lim _{u \rightarrow+\infty} i\left(\alpha, m_{u}\right) E_{\alpha_{1}}^{t}\left(\frac{1}{i\left(\alpha, m_{u}\right)} \frac{1}{u} E_{\alpha_{2}}^{t u i\left(\alpha, m_{u}\right)+u} E_{\beta_{1}}^{u} m\right) \\
& =t^{-1} E_{\alpha_{1}}^{t} \frac{1}{t^{-1}}\left(2 \alpha_{2} \cup \beta_{1}\right)=E_{\alpha_{1}}^{1}\left(2 \alpha_{2} \cup \beta_{1}\right)=2 \alpha_{2} \cup E_{\alpha_{1}}^{1} \beta_{1} .
\end{aligned}
$$

On the other hand, similar computations give that

$$
T_{\alpha}^{t} m_{u}^{\prime}=E_{\alpha_{1}}^{t i\left(\alpha, m_{u}^{\prime}\right)}\left(\frac{1}{u} E_{\alpha_{2}}^{t u i\left(\alpha, m_{u}^{\prime}\right)-u} E_{\beta_{1}}^{-u} m\right) .
$$

As above, an application of Lemma 16 shows that

$$
\begin{aligned}
& \left|i\left(\frac{1}{u} E_{\alpha_{2}\left(\alpha, m_{u}^{\prime}\right)-u}^{t u i} E_{\beta_{1}}^{-u} m, \gamma\right)-i\left(\frac{1}{u} E_{\beta_{1}}^{-u} m, \gamma\right)\right| \\
& \quad \leq \frac{1}{u}\left|t u i\left(\alpha, m_{u}^{\prime}\right)-u\right| i\left(\gamma, \alpha_{2}\right) \\
& \quad \leq\left|t i\left(\alpha, m_{u}^{\prime}\right)-1\right| i\left(\gamma, \alpha_{2}\right) \rightarrow 0
\end{aligned}
$$

for every simple closed geodesic $\gamma$, and therefore that

$$
\lim _{u \rightarrow+\infty} \frac{1}{u} E_{\alpha_{2}}^{t u i\left(\alpha, m_{u}^{\prime}\right)-u} E_{\beta_{1}}^{-u} m=\lim _{u \rightarrow+\infty} \frac{1}{u} E_{\beta_{1}}^{-u} m=\beta_{1} .
$$

By Theorem 14, it follows that

$$
\begin{aligned}
\lim _{u \rightarrow+\infty} T_{\alpha}^{t} m_{u}^{\prime} & =\lim _{u \rightarrow+\infty} E_{\alpha_{1}}^{t i\left(\alpha, m_{u}^{\prime}\right)}\left(\frac{1}{u} E_{\alpha_{2}}^{t u i\left(\alpha, m_{u}^{\prime}\right)-u} E_{\beta_{1}}^{-u} m\right) \\
& =\lim _{u \rightarrow+\infty} i\left(\alpha, m_{u}^{\prime}\right) E_{\alpha_{1}}^{t}\left(\frac{1}{i\left(\alpha, m_{u}^{\prime}\right)} \frac{1}{u} E_{\alpha_{2}}^{t u i\left(\alpha, m_{u}^{\prime}\right)-u} E_{\beta_{1}}^{-u} m\right) \\
& =t^{-1} E_{\alpha_{1}}^{t}\left(\frac{1}{t^{-1}} \beta_{1}\right)=E_{\alpha_{1}}^{1} \beta_{1} .
\end{aligned}
$$

Therefore, $T_{\alpha}^{t} m_{u}$ and $T_{\alpha}^{t} m_{u}^{\prime}$ have different limits although $m_{u}$ and $m_{u}^{\prime}$ both converge to $\beta$. This proves that $T_{\alpha}^{t}$ cannot extend continuously to $\mathscr{T}_{10, \infty]}(S)$ if $\alpha$ is not connected.

\section{LENGTHS OF MEASURED LAMINATIONS AND OF GEODESIC CURRENTS}

In $\S 3$ we gave a formula for the derivative $\left.\frac{\partial}{\partial t^{+}} i\left(E_{\alpha}^{t} \mu, \gamma\right)\right|_{t=0}$ when $\mu \epsilon$ $\mathscr{T}_{10, \infty]}(S)$ and when $\gamma$ is a simple closed geodesic. We want to extend this formula to the case where $\gamma$ is a measured geodesic lamination or, better, when $\gamma$ is a geodesic current in the sense of [Bo1, Bo2].

When $\gamma$ is a simple closed geodesic, we proved in $\S 3$ that

$$
i\left(E_{\alpha}^{t} \mu, \gamma\right)-i(\mu, \gamma)=\int_{0}^{t} \int_{\gamma} \cos _{E_{\alpha}^{\mu} \mu}^{+}(\gamma, \alpha) d \alpha d u .
$$

Actually, inspecting the proof of Propositions 5 and 6, we can observe that we did not use the fact that $\gamma$ was simple. The above equality therefore holds for 
any closed geodesic (possibly with self-intersection points). We will extend it to the case where $\gamma$ is a geodesic current, first making sense of the right-hand side of the equality in that case.

Before that, let us recall a few definitions, referring to [Bo1, $\S 4$; Bo2] for more details. Endow $S$ with an arbitrary negatively curved metric $m_{0}$, and consider the space $G(\widetilde{S})$ of $m_{0}$-geodesics of the universal covering $\widetilde{S}$ of $S$. Up to homeomorphism preserving the natural action of the fundamental group $\pi_{1}(S)$, this space turns out to be independent of the choice of the metric $m_{0}$. A geodesic current is a (positive Radon) $\pi_{1}(S)$-invariant measure on the space $G(\widetilde{S})$. A typical example is associated to each closed geodesic; indeed, the lifts of this geodesic define a $\pi_{1}(S)$-invariant discrete subset of $G(\widetilde{S})$, and the Dirac (counting) measure defined by this discrete subset is a geodesic current. This example is fairly general, since the positive real multiples of geodesic currents so associated to closed geodesics are dense in the space $\mathscr{C}(S)$ of all geodesic currents on $S$. Thus, exactly like a measured geodesic lamination is some kind of diffuse simple closed geodesic, a geodesic current is some kind of diffuse closed geodesic. The embedding of the set of simple closed geodesics in the set of all closed geodesics extends to an embedding of the space $\mathscr{M} L(S)$ of measured laminations in the space $\mathscr{C}(S)$ of geodesic currents. This embedding is continuous if $\mathscr{C}(S)$ is endowed with the weak* topology (as a space of measures; see [Bou, Chapter III, $\S 1]$ or [Be] for instance).

There is a well-defined continuous geometric intersection number $i(\alpha, \beta)$ of two geodesic currents $\alpha$ and $\beta$, which extends the one of Proposition 3. To define it, let $D G(\widetilde{S})$ be the subset of $G(\widetilde{S}) \times G(\widetilde{S})$ consisting of those pairs of geodesics $(g, h)$ which meet transversely in one point. The group $\pi_{1}(S)$ acts freely and properly discontinuously on $D G(\widetilde{S})$, and we can consider the quotient $D G(S)=D G(\widetilde{S}) / \pi_{1}(S)$. The two geodesic currents define a product measure $\alpha \times \beta$ on $G(\widetilde{S}) \times G(\widetilde{S})$, and therefore on $D G(\widetilde{S})$ and $D G(S)$. Then, by definition, the geometric intersection number of $\alpha$ and $\beta$ is the total mass $i(\alpha, \beta)$ of this measure $\alpha \times \beta$ on $D G(S)$, namely

$$
i(\alpha, \beta)=\int_{D G(S)} d(\alpha \times \beta) .
$$

An argument shows that this integral is finite, and that the map $(\alpha, \beta) \mapsto$ $i(\alpha, \beta)$ so defined is continuous on $\mathscr{C}(S) \times \mathscr{C}(S)$. When $\alpha$ and $\beta$ are associated to two distinct closed geodesics, $i(\alpha, \beta)$ is exactly equal to the number of intersection points of these two geodesics.

Now, given $\mu \in \mathscr{T}_{30, \infty]}(S)$ and $(g, h) \in D G(\widetilde{S})$, consider the $\mu$-cosine $\cos _{\mu}^{+}(g, h)$ defined as in $\S 3$. This function is clearly $\pi_{1}(S)$-invariant and descends to a function $D G(S) \rightarrow \mathbb{R}^{+}$. For $\alpha, \beta \in \mathscr{C}(S)$ we can then consider the integral

$$
\int_{D G(S)} \cos _{\mu}^{+}(g, h) d(\alpha \times \beta)(g, h) .
$$

The proof that this integral is finite is similar to the proof of the finiteness of $i(\alpha, \beta)$ given in [Bo1, §4]. (The key point is that $\left|\cos _{\mu}^{+}(g, h)\right| \leq 1$.)

When the geodesic currents $\alpha, \beta \in \mathscr{C}(S)$ correspond to closed geodesics, it is immediate that the above integral is just the sum of the $\cos _{\mu}^{+}(\alpha, \beta)(x)$ 
(using the notation of $\S 3$ ), where $x$ ranges over all intersection points of these geodesics. Similarly, if $\gamma \in \mathscr{C}(S)$ corresponds to a closed geodesic and $\alpha \in$ $\mathscr{C}(S)$ corresponds to a measured geodesic lamination,

$$
\int_{D G(S)} \cos _{\mu}^{+}(g, h) d(\gamma \times \alpha)(g, h)=\int_{\gamma} \cos _{\mu}^{+}(\gamma, \alpha) d \alpha,
$$

which is exactly the formula occurring in the expression of $\left.\frac{\partial}{\partial t^{+}} i\left(E_{\alpha}^{t} \mu, \gamma\right)\right|_{t=0}$.

Proposition 20. If $\mu \in \mathscr{T}_{10, \infty[}(S)$, the map

$$
(\alpha, \beta) \mapsto \int_{D G(S)} \cos _{\mu}^{+}(g, h) d(\alpha \times \beta)(g, h)
$$

is continuous on $\mathscr{C}(S) \times \mathscr{C}(S)$. If $\mu \in \mathscr{T}_{\infty}(S)$, it is continuous at those $(\alpha, \beta) \in$ $\mathscr{C}(S) \times \mathscr{C}(S)$ for which the subset of $G(\widetilde{S})$ consisting of the geodesics of $\widetilde{S}$ whose projection to $S$ is contained in the support of $\mu$ has $\alpha$-mass and $\beta$-mass equal to 0 .

Proof. The proof splits into a local part and a global part. First, for $\varepsilon>0$, we want to find a compact subset $K$ of $D G(S)$ such that

$$
\left|\int_{D G(S)-K} \cos _{\mu}^{+}(g, h) d\left(\alpha^{\prime} \times \beta^{\prime}\right)(g, h)\right|<\varepsilon
$$

for all $\left(\alpha^{\prime}, \beta^{\prime}\right) \in D G(S)$ sufficiently close to $(\alpha, \beta)$. Then, for such a $K$, we need to show that

$$
\int_{K} \cos _{\mu}^{+}(g, h) d\left(\alpha^{\prime} \times \beta^{\prime}\right)(g, h)
$$

converges to

$$
\int_{K} \cos _{\mu}^{+}(g, h) d(\alpha \times \beta)(g, h)
$$

as $\left(\alpha^{\prime}, \beta^{\prime}\right)$ converges to $(\alpha, \beta)$.

Finding such a $K$ is done as in the proof of the continuity of $i(\alpha, \beta)$ in [Bo1, §4]. (Again the key point is that $\cos _{\mu}^{+}(g, h) \leq 1$.) The $K$ provided by this proof also has the additional property that its boundary has $(\alpha \times \beta)$-mass equal to 0 .

If $\mu \in \mathscr{T}_{10, \infty[}$, the function $(g, h) \mapsto \cos _{\mu}^{+}(g, h)$ is continuous everywhere. Since the boundary of $K$ has $(\alpha \times \beta)$-mass 0 , it follows that

$$
\int_{K} \cos _{\mu}^{+}(g, h) d\left(\alpha^{\prime} \times \beta^{\prime}\right)(g, h)
$$

converges to $\int_{K} \cos _{\mu}^{+}(g, h) d(\alpha \times \beta)(g, h)$ as the measure $\left(\alpha^{\prime} \times \beta^{\prime}\right)$ converges to $(\alpha \times \beta)$ for the weak ${ }^{*}$ topology (see [Bou, Chapter IV, §5] or [Be, Chapter III], for instance), and therefore as $\left(\alpha^{\prime}, \beta^{\prime}\right)$ converges to $(\alpha, \beta)$ in $\mathscr{C}(S) \times \mathscr{C}(S)$.

If $\mu \in \mathscr{T}_{\infty}(S)$, the discontinuities of the function $(g, h) \mapsto \cos _{\mu}^{+}(g, h)$ occur at those $(g, h)$ where one of $g$ or $h$ projects to a geodesic contained in the support of $\mu$. By hypothesis on $\alpha$ and $\beta$, the set of these discontinuities has $(\alpha \times \beta)$-mass 0 . It again follows that $\int_{K} \cos _{\mu}^{+}(g, h) d\left(\alpha^{\prime} \times \beta^{\prime}\right)(g, h)$ converges to $\int_{K} \cos _{\mu}^{+}(g, h) d(\alpha \times \beta)(g, h)$ as $\left(\alpha^{\prime}, \beta^{\prime}\right)$ converges to $(\alpha, \beta)$ for the weak ${ }^{*}$ topology. This concludes the proof of Proposition 20. 
Proposition 21. For $\mu \in \mathscr{T}_{00, \infty]}(S), \alpha \in \mathscr{M} L(S)$, and $\beta \in \mathscr{C}(S)$,

$$
i\left(E_{\alpha}^{t} \mu, \beta\right)-i(\mu, \beta)=\int_{0}^{t} \int_{D G(S)} \cos _{E_{\alpha}^{u} \mu}^{+}(g, h) d(\beta \times \alpha)(g, h) d u .
$$

Proof. As observed above, we proved this formula in $\S 3$ when $\beta=\gamma$ is a simple closed curve. By homogeneity, it also holds when $\beta$ is a positive real multiple of a closed geodesic. We will use a continuity argument to prove it in the general case.

Since (positive real) multiples of closed geodesics are dense in $\mathscr{C}(S)$, we can write $\beta \in \mathscr{C}(S)$ as a limit of multiples of closed geodesics $\gamma_{i} \in \mathscr{C}(S)$.

Since we intend to use Proposition 20, we can expect some complications when $\mu \in \mathscr{T}_{\infty}(S)$. Let us make some preliminary observations in this case. First of all, we can assume without loss of generality that every component of $\alpha$ transversely meets $\mu$ and that every component of $\mu$ transversely meets $\alpha$; indeed, subtracting the components of $\alpha$ and $\mu$ which do not cross the other geodesic lamination does not change either side of the equality. Under this condition, let us come back to the definition of $E_{\alpha}^{u} \mu$ in $\S 2$, using measured foliations. In that construction, $E_{\alpha}^{u} \mu$ and $E_{\alpha}^{v} \mu$ are represented by measured foliations $E_{A}^{u} M$ and $E_{A}^{v} M$ such that, if $u \neq v$, every leaf of $E_{A}^{u} M$ is transverse to $E_{A}^{v} M$. It easily follows that, if $u \neq v$, the supports of $E_{\alpha}^{u} \mu$ and $E_{\alpha}^{v} \mu$ never have a leaf in common. As a consequence, there are at most countably many values of $u$ for which the subset of those geodesics of $\widetilde{S}$ whose projection to $S$ is contained in the support of $E_{\alpha}^{u} \mu$ has positive $\beta$-mass.

We are now ready to prove Proposition 21. By Proposition 20 and by the above observation (assuming that, when $\mu \in \mathscr{T}_{\infty}(S)$, each component of $\alpha$ crosses $\mu$ and that each component of $\mu$ crosses $\alpha$ ),

$$
\int_{D G(S)} \cos _{E_{\alpha}^{\mu} \mu}^{+}(g, h) d\left(\gamma_{i} \times \alpha\right)(g, h)
$$

converges to $\int_{D G(S)} \cos _{E_{\alpha}^{\mu} \mu}^{+}(g, h) d(\beta \times \alpha)(g, h)$ for all but (at most) countably many values of $u$. We conclude that

$$
\int_{0}^{t} \int_{D G(S)} \cos _{E_{\alpha}^{\mu} \mu}^{+}(g, h) d\left(\gamma_{i} \times \alpha\right)(g, h) d u
$$

converges to $\int_{0}^{t} \int_{D G(S)} \cos _{E_{\alpha}^{\mu} \mu}^{+}(g, h) d(\beta \times \alpha)(g, h) d u$.

On the other hand,

$$
\int_{0}^{t} \int_{D G(S)} \cos _{E_{\alpha}^{u} \mu}^{+}(g, h) d\left(\gamma_{i} \times \alpha\right)(g, h) d u=i\left(E_{\alpha}^{t} \mu, \gamma_{i}\right)-i\left(\mu, \gamma_{i}\right) .
$$

It follows that $\int_{0}^{t} \int_{D G(S)} \cos _{E_{\alpha}^{\mu} \mu}^{+}(g, h) d(\beta \times \alpha)(g, h) d u$ is equal to the limit of $i\left(E_{\alpha}^{t} \mu, \gamma_{i}\right)-i\left(\mu, \gamma_{i}\right)$, namely to $i\left(E_{\alpha}^{t} \mu, \beta\right)-i(\mu, \beta)$ by continuity of the intersection number. This concludes the proof.

By analogy with the definition introduced in $\S 4$, we will say that $\beta \in \mathscr{T}_{\infty}(S)$ spans $(g, h) \in D G(S)$ if, when we lift $(g, h)$ to $(\tilde{g}, \tilde{h}) \in D G(\widetilde{S})$ and $\beta$ to a measured geodesic lamination $\widetilde{\beta}$ of $\tilde{S}$, there is at least one leaf of $\widetilde{\beta}$ which transversely cuts both $\widetilde{g}$ and $\widetilde{h}$.

We now have the following analog of Proposition 6 (with some more restricted hypotheses). 
Corollary 22. If $\mu \in \mathscr{T}_{10, \infty[}(S)$, or if $\mu$ is in $\mathscr{T}_{\infty}(S)$ and spans $(\alpha \times \beta)$-almost all $(g, h) \in D G(S)$ then, for every $\alpha \in \mathscr{M} L(S)$ and $\beta \in \mathscr{C}(S)$,

$$
\left.\frac{\partial}{\partial t} i\left(E_{\alpha}^{t} \mu, \beta\right)\right|_{t=0}=\int_{D G(S)} \cos _{\mu}^{+}(g, h) d(\beta \times \alpha)(g, h) .
$$

Proof. Consider the integral

$$
\varphi(u)=\int_{D G(S)} \cos _{E_{\alpha}^{\mu} \mu}^{+}(g, h) d(\beta \times \alpha)(g, h) .
$$

As $u$ tends to $0, E_{\alpha}^{u} \mu$ converges to $\mu$ and, by hypothesis on $\mu$, it follows that $\cos _{E_{\alpha}^{\mu} \mu}^{+}(g, h)$ converges to $\cos _{\mu}^{+}(g, h)$ for $(\alpha, \beta)$-almost all $(g, h) \in$ $D G(S)$. By dominated convergence, this implies that $\varphi$ is continuous at 0 . We conclude that the $t$-derivative of $i\left(E_{\alpha}^{t} \mu, \beta\right)=i(\mu, \beta)+\int_{0}^{t} \varphi(u) d u$ at 0 is equal to $\varphi(0)$.

Observe that we needed a hypothesis in Corollary 22 which did not appear in Proposition 6, namely the condition that, when $\mu \in \mathscr{T}_{\infty}(S)$, it spans $(\alpha \times \beta)$-almost all $(g, h) \in D G(\widetilde{S})$. This condition was required by the continuity argument which we used in our proof. Presumably, this technical hypothesis is unnecessary and the cosine formula for $\left.\frac{\partial}{\partial t^{+}} i\left(E_{\alpha}^{t} \mu, \beta\right)\right|_{t=0}$ holds without this restriction.

One case of interest is when the measured geodesic lamination $\mu \in \mathscr{T}_{\infty}(S)$ is complete, namely when the complement of the support of $\mu$ in $S$ consists of (infinite) triangles. Then the only $(g, h) \in D G(S)$ which are not spanned by $\mu$ are those for which $g$ or $h$ projects to a leaf in the support of $\mu$. In particular, the hypothesis of Corollary 22 is then satisfied if $\alpha$ and $\beta$ are measured geodesic laminations with support different from the support of $\mu$.

One reason to be interested in the derivative $\left.\frac{\partial}{\partial t^{t}} i\left(E_{\alpha}^{t} \mu, \beta\right)\right|_{t=0}$ comes from the symplectic structures of the spaces $\mathscr{T}_{k}(S)$.

Indeed, Teichmüller space $\mathscr{T}(S)$ is a complex manifold and possesses a natural Kähler form called the Weil-Petersson form. The real part of this Kähler form defines a symplectic structure on $\mathscr{T}_{k}(S)$ for each $\left.k \in\right] 0, \infty[$, namely induces an antisymmetric real-valued bilinear form $\omega$ on each tangent space to $\mathscr{T}_{k}(S)$.

On the other hand, $\mathscr{T}_{\infty}(S)$ does not admit any differentiable structure that is respected by the action of the diffeomorphisms of $S$. Instead, $\mathscr{T}_{\infty}(S)$ has a natural structure of piecewise linear manifold. At a $\mu \in \mathscr{T}_{\infty}(S)$ which is complete measured geodesic lamination, this piecewise linear structure is however sufficiently nice to define a linear tangent space to $\mathscr{T}_{\infty}(S)$ at this point, and Thurston introduced a symplectic form $\omega$ on this linear tangent space (see [Pa2, $\mathrm{Pa} 3])$.

When such a linear tangent space exists, namely when $\mu \in \mathscr{T}_{k}(S)$ with $k \in$ ]0, $\infty\left[\right.$ or when $\mu \in \mathscr{T}_{\infty}(S)$ is complete, the derivative $\left.\frac{\partial}{\partial t} E_{\alpha}^{t} \mu\right|_{t=0}$ defines a tangent vector to $\mathscr{T}_{k}(S)$ at $\mu$. In addition, these earthquake derivatives linearly generate this tangent space. Also, if $\alpha, \beta \in \mathscr{M} L(S)$, the symplectic structure $\omega$ is such that

$$
\omega\left(\left.\frac{\partial}{\partial t} E_{\alpha}^{t} \mu\right|_{t=0},\left.\frac{\partial}{\partial t} E_{\beta}^{t} \mu\right|_{t=0}\right)=\left.\frac{\partial}{\partial t} i\left(E_{\alpha}^{t} \mu, \beta\right)\right|_{t=0}
$$

(see [Wo2]). 
In this context, Proposition 12 suggests that, as $m \in \mathscr{T}_{k(m)}(S)$ tends to a complete $\mu \in \mathscr{T}_{\infty}(S)$, the linear tangent space to $\mathscr{T}_{k(m)}(S)$ at $m$ converges to the linear tangent space of $\mathscr{T}_{\infty}(S)$ at $\mu$. Also, by dominated convergence, Corollary 22 and Lemma 11 indicate that the symplectic structures $\omega$ vary continuously in this convergence.

\section{REFERENCES}

[Be] H. Bergström, Weak convergence of measures, Academic Press, New York, 1982.

[Bo1] F. Bonahon, Bouts des variétés hyperboliques de dimension 3, Ann. of Math. (2) 124 (1986), 71-158.

[Bo2] _ The geometry of Teichmüller space via geodesic currents, Invent. Math. 92 (1988), 139-162.

[Bou] N. Bourbaki, Éléments de mathématiques. Livre VI (Intégration), Hermann, Paris, 1965.

[CB] A. Casson and S. A. Bleiler, Automorphisms of surfaces after Nielsen and Thurston, Cambridge Univ. Press, 1988.

[EM] D. B. A. Epstein, and A. Marden, Convex hulls in hyperbolic space, a theorem of Sullivan, and measured pleated surfaces, Analytical and Geometric Aspects of Hyperbolic Space (D. B. A. Epstein, ed.), Cambridge Univ. Press, 1986, pp. 113-253.

[Fal] A. Fathi, Dehn twists and pseudo-Anosov diffeomorphisms, Invent. Math. 87 (1987), 129151.

[Fa2] , The Poisson bracket on the space of measured foliations on a surface, Duke Math. J. 55 (1987), 693-697.

[FLP] A. Fathi, F. Laudenbach, and V. Poenaru, Travaux de Thurston sur les surfaces, Astérisque, no. 66-67, Soc. Math. France, 1979.

[FN] W. Fenchel and J. Nielsen, Discontinuous groups of non-Euclidean motions, unpublished manuscript.

[Ke] S. P. Kerckhoff, The Nielsen realization problem, Ann. of Math. (2) 117 (1983), 235-265.

[Le] G. Levitt, Foliations and laminations on hyperbolic surfaces, Topology 22 (1983), 119-135.

[Pa1] A. Papadopoulos, L'extension du flot de Fenchel-Nielsen au bord de Thurston de l'espace de Teichmüller, C. R. Acad. Sci. Paris Sér. I Math. 302 (1986), 325-327.

[Pa2] _ Deux remarques sur la géométrie symplectique de l'espace des feuilletages mesurés sur une surface, Ann. Inst. Fourier (Grenoble) 36 (1986), 127-141.

[Pa3] $\_$, Geometric intersection functions and Hamiltonian flows on the space of measured foliations of a surface, Pacific J. Math. 124 (1986), 375-402.

[Pa4] On Thurston's boundary of Teichmüller space and the extension of earthquakes, Topology Appl. (to appear).

[Re] M. Rees, An alternative approach to the ergodic theory of measured foliations on surfaces, Ergodic Theory Dynamical Systems 1 (1981), 461-488.

[Th1] W. P. Thurston, On the geometry and dynamics of diffeomorphisms of surfaces, Bull. Amer. Math. Soc. (N.S.) 19 (1988), 417-431.

[Th2] _ The geometry and topology of 3-manifolds, lecture notes, Princeton University, 1976-1979.

[Th3] _ Earthquakes in two-dimensional hyperbolic geometry, Low-Dimensional Topology and Kleinian Groups (D. B. A. Epstein, ed.), Cambridge Univ. Press, 1986, pp. 91-112.

[Wo1] S. Wolpert, The Fenchel-Nielsen deformation, Ann. of Math. (2) 115 (1982), 501-528.

[Wo2] _ On the symplectic geometry of deformations of a hyperbolic surface, Ann. of Math. (2) 117 (1983), 207-234.

Department of Mathematics, University of Southern California, Los Angeles, CaliFORNIA 90089-1113 